\title{
KNOWLEDGE OF ETHNOMEDICAL PLANTS AND INFORMANT CONSENSUS IN AND AROUND LAWACHARA NATIONAL PARK
}

\author{
MOHAMMAD ZASHIM UDDIN ${ }^{1}$, MD. KAMRUL AREFIN, MD. FAKHRUL ALAM, MD. \\ GOLAM KIBRIA, SANKAR LAL PODDER AND MD. ABUL HASSAN \\ Department of Botany, University of Dhaka, Dhaka-1000, Bangladesh
}

\begin{abstract}
Consensus of the people's healthcare knowledge of ethno-medicinal plants in and around Lawachara national park was conducted from December 2014 to November 2015. The main aim of the study was to record and document plants species used for the treatment of various ailments and to find out level of consensus and agreement between informants regarding uses of plant for particular ailment categories. Data of medicinal uses of plants were recorded through semi-structured interviews, key informant discussions and informal conversations with local and ethnic people including herbal practitioners. A total of 124 medicinal plant species with 245 formularies to treat 53 ailments were recorded. For each species scientific name, local name, family, part used, ailments to be treated and mode of treatment are presented. Leaf is the dominant part used followed by fruit, root and rhizome, whole plant, seed, stem, bark, petiole, bulb, peduncle, latex and flower. In the documented 124 species, herbs were represented by $43 \%$, trees by $31 \%$, shrubs by $15 \%$ and climbers by $11 \%$ species. Oral consumption is the main mode of treatment in the study area and followed by external application. Maximum formularies were found in the six ailment groups including gastrointestinal complain, diarrhoea and dysentery, fever and cough, dermatitis, jaundice and impotence. According to the local people most of the plants (59\%) were harvested from the park vegetation and minimum $(41 \%)$ harvested from cultivated source. Maximum consensus values (Factor Informants Consensus (Fic) values more than 0.80) were obtained in case of cut and wounds and followed by Jaundice, Respiratory related ailments, Diabetes, Diarrhoea and dysentery, Anthelmintic, Gastrointestinal complain, Impotence, Dermatitis and High blood pressure. Chromolaena odoratum, Cericoides campanulata, Oroxylum indicum, Cuscuta reflexa, Averrhoa carambola, Cajanus cajan, Justicia adhatoda and Citrus aurantifolia showed 100\% Fidelity values (Fl). Litsea glutinosa, Mikania cordata, Ocimum sanctum and Azadirachta indica were scored maximum Percent of Respondents knowledge values (PRK). It is recommended that species which showed high Fic, Fl and PRK values could be used for further ethno-lead phytochemical analysis to investigate active compounds to discover drugs from plants. Finally, a number of threats to medicinal plants were identified and some measures for conservation are also suggested.
\end{abstract}

Key words: Consensus, Healthcare, Ethno-medicinal, Lawachara National Park

\footnotetext{
${ }^{1}$ Corresponding author: zashim01@gmail.com
} 


\section{Introduction}

Consensus of the people's in the use of healthcare scientific knowledge of ethnomedicinal plants is the gateway in identifying new plant products of potential and commercial values. It is estimated that there are 250,000 to 500,000 species of plants on Earth (Borris 1999). A relatively small percentage (1 to 10\%) of these is used as food by both humans and other animal species. It is possible that even more are used for medicinal purposes (Moerman 1996). Hippocrates (in the late fifth century B.C.) mentioned 300 to 400 medicinal plants (Schultes 1978). In the first century A.D., Dioscorides wrote De Materia Medica, a medicinal plant catalog which became the prototype for modern pharmacopoeias. Documented medicinal plants with high degree of consensus can serve as a basis for future investigation of modern drug (Khan et al. 2014). Plant based traditional medicine plays a key role in the development of novelties in drug discovery (Wright 2005). Recent studies showed that over $80 \%$ rural people of the world rely on herbal medicines (Setzer et al. 2006). The world market for herbal medicines based on traditional knowledge is now estimated at US\$ 60 billion (Breevot 1998). World leaders met in Rio de Janeiro during 1992 to formulate biodiversity conservation policy including agenda 21 which also gave emphasis on the documentation and sustainable utilization of traditional knowledge of medicinal plants.

Currently, Ethnomedicinal knowledge of plants has been eroding at alarming rate from the nature before proper documentation and evaluation. In order to protect such knowledge, documentation of ethnomedicinal plants has already been started in Bangladesh. A number of articles was published in this field including Mia and Huq (1988), Hassan and Khan (1986, 1996), Alam (1992), Alam et al. (1996), Uddin (2006), Uddin et al. (2001), Khan et al. (2002), Yusuf et al. (2002), Uddin et al. (2004), Uddin et al. (2006), Yusuf et al. (2006), Uddin and Roy (2007), Uddin et al. (2008), Uddin et al. 2012, Haque et al. (2014) and Uddin and Hassan (2014). All such articles were listed a good number of medicinal plants of particular community or particular diseases or particular areas of Bangladesh. But there are still more medicinal plants used as sources of herbal drugs by the ethnic and local people of Bangladesh yet to be discovered. Unfortunately no such works have covered the quantitative documentation of ethnomedicinal plants of rural people living in and around Lawachara national park. The park supports a large number of plant species. Among them many species are medicinal those need to be documented and conserved. Local people in and around the park had been using such plants in their primary health care. Currently medicinal plants and traditional knowledge have been eroding due to globalization and climate change. Some of such knowledge is going to eliminate before documentation which is alarming to sustain cultural heritage. In order to save the healthcare knowledge and medicinal plants, in the present study an attempt was made to achieve the following objectives: to record, integrate and document all scattered distribution of traditional healthcare knowledge of medicinal plants; to determine ethno-medically potential and culturally important and 
most cited medicinal plants using statistical models; to find out the threats to medicinal plants in the natural habitats and to suggest measures for the conservation

\section{Materials and Methods}

Lawachara national park, under Kamalganj upazila of Maulvi Bazar district, is a part of West Bhanugach reserve forest, which was declared reserve in early nineteenth century as per the Forest Act 1878, the Assam Forest Manual 1898 and the Forest Act 1927 (USAID 2006). The park is located nearly $160 \mathrm{~km}$ northeast of Dhaka and approximately $60 \mathrm{~km}$ south of Sylhet city (Map 1).It lies between $24^{\circ} 30^{\prime}-24^{\circ} 32^{\prime} \mathrm{N}$ and $91^{\circ} 37^{\prime}-91^{\circ} 39^{\prime} \mathrm{E}$. The forest was declared as a national Park in 1996 having a total area of 1250 ha and with a plan to extend this area further to include 281 additional ha of the Reserve Forest (Green 1990, Canonizado and Rahman 1998, Riadh 2007 and Ahsan 2007).

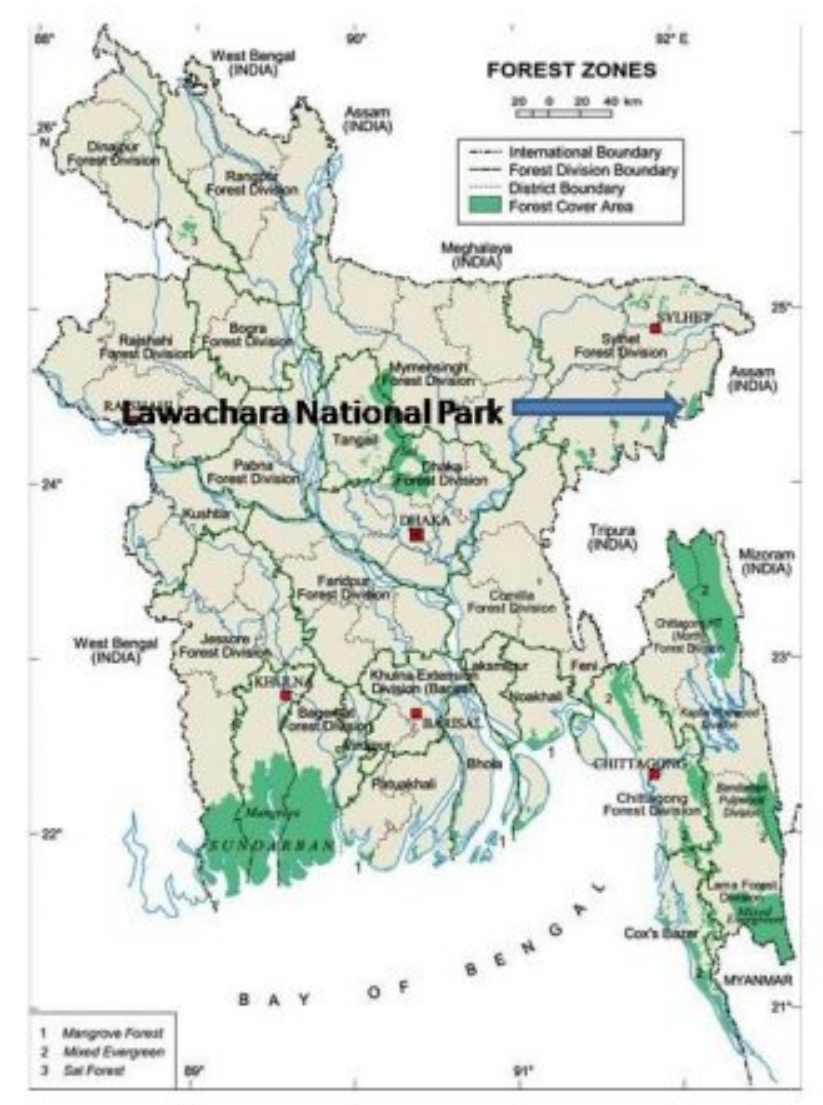

Map 1 (a). Bangladesh Map showing Lawachara National Park. 


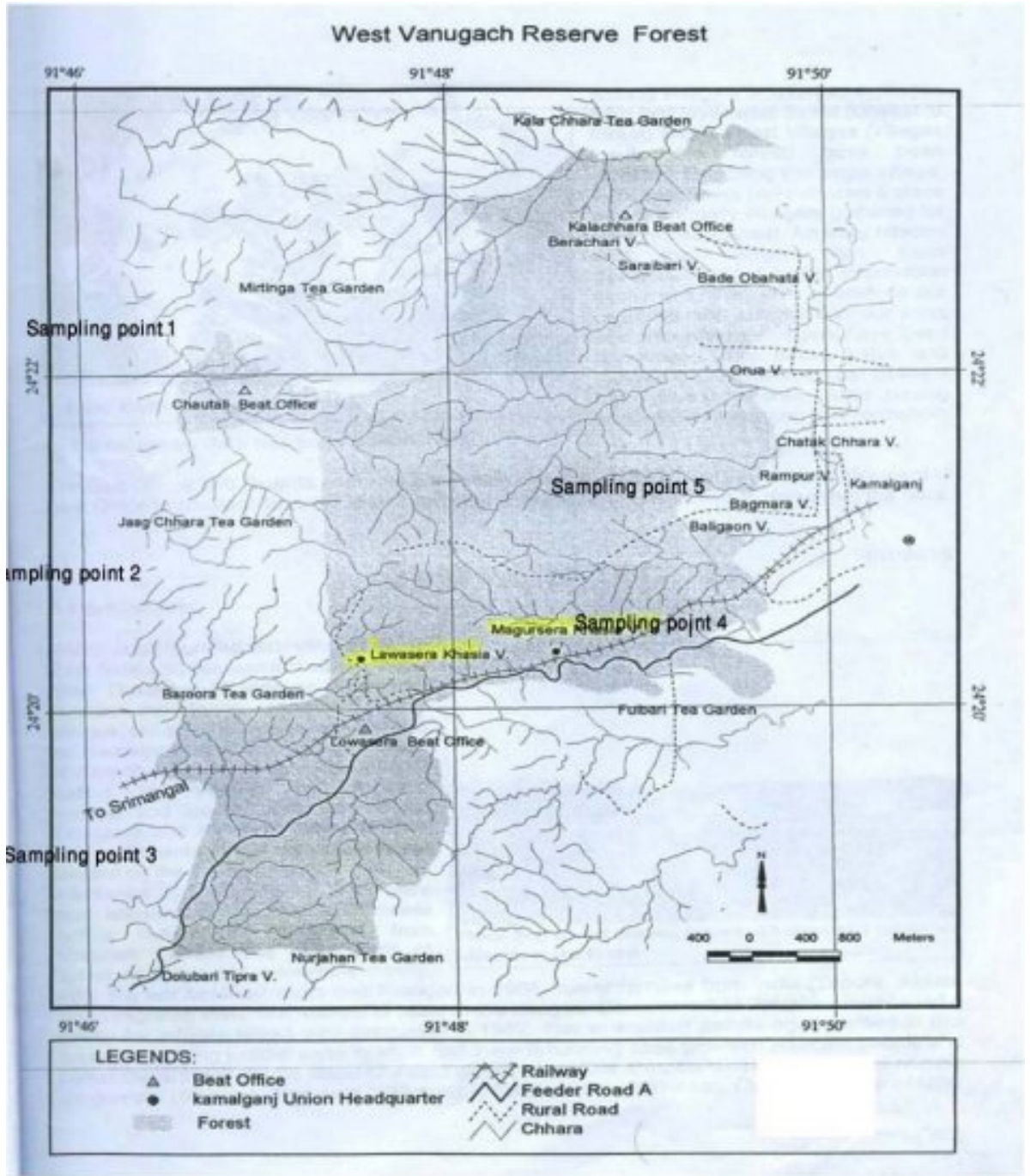

Map 1. (b) Enlarged part showing different sampling points of Lawachara National Park.

(Source: Collected from Web).

Present forest types of Lawachara are a combination of planted exotic species and mixed forest with a deciduous canopy and an evergreen understory (Ahsan 2000). The forest originally supported an indigenous vegetation cover of mixed tropical evergreen type (Alam 1998). The topography of Lawachara National Park is undulating, with slopes and hillocks that range from 10 to $50 \mathrm{~m}$ in elevation (Rizvi 1970 and Riadh 2007). These hillocks are scattered and interspersed with numerous streams that flow through the forest. The hills are composed of upper tertiary rocks in which sand stone largely 
predominates (Ahmad 1970 and Stevens 1986) along with siltstones and mudstones, locally altered to slates and shales. The significant soils in the hills of Maulovi Bazar belong to Ramgarh and Rangamati series on Dupitila formation (Stevens 1986). Soils of the park are generally sandy loam and the rest are mostly clayey loam (Ahmad 1970). The area enjoys a moist tropical climate characterized by a period of high precipitation from April to September and five months of relatively dry period from November to March.

\section{Methods of data collection}

The study area has been visited four times in different seasons of the year of 2015 including summer, rain, autumn and winter. Each field trip lasted for five days. The data of medicinal uses were recorded through semi-structured interviews, key informant discussions and informal conversations with local people including herbal practitioners (Alexiades 1996, Chambers 1994 and Martin 1995). A total of 163 informants was interviewed using questionnaire. Among them 117 male and rest 46 are female. Age ranges from 15 to 95 years old. Education levels of the informants were from illiterate up to Bachelor degree. Professionally they were mostly farmer, day labor, house wife, small shopkeepers and medicine men. During the field survey, information on uses of plants to treat human, parts used, mode of preparation and administration was documented. The vernacular names were collected with the help of local people. Voucher specimens for each medicinal plant were collected and processed using standard herbarium techniques (Hyland 1972 and Alexiades 1996). The specimens were identified consulting different Floras viz., Hooker 1872-1897, Prain 1903, Uddin and Hassan 2004, Siddiqui et al. 2007c and Ahmed et al. 2008a, 2008b, 2009b, 2009c, 2009d and 2009e. Specimens available at Dhaka University Salar Khan Herbarium (DUSH) were consulted in identifying the collected plant specimens. The updated nomenclature of the species followed Siddiqui et al. 2007c and Ahmed et al. 2008a, 2008b, 2009b, 2009c, 2009d, and 2009e. Voucher specimens are preserved at DUSH.

\section{Factor of informant consensus}

In order to estimate use diversity of the medicinal plants and to determine which plants are particularly interesting in the search for bioactive compounds, factor of informant consensus (Fic) was calculate (Trotter and Logan 1986 and Heinrich et al. 1998).

Fic is thus calculated applying the following equation: Fic $=$ Nur - Ntaxa/Nur-1

Where Nur is the number of use reports in each category, Ntaxa is the number of species in each category. The relative importance of a species is evaluated by the proportion of 
respondents who cited it. The Fic provides a range of $0-1$, where high values (close to 1) are obtained when only one or a few plant species are reported to be used by a high proportion of informants to treat a particular ailment. High Fic thus means that there is a narrow well-defined group of species used to cure a particular ailment category and/or that information is exchanged between informants. On the other hand, low Fic values (close to zero) indicate that informants disagree over which plant to use due to random choosing or lack of exchange of information about use among informant.

\section{Fidelity level (FI)}

The Fl value is useful for identifying the informants most preferred species in use for treating certain ailments (Friedman et al.1986). the $\mathrm{Fl}$ index, $\mathrm{Fl}=\mathrm{I}_{\mathrm{p}} / \mathrm{I}_{\mathrm{u}} \mathrm{x} 100$, where $\mathrm{I}_{\mathrm{p}}$ is number of informants who indicate use of a species for the same major ailment, $\mathrm{I}_{\mathrm{u}}$ is the total number of informants who mentioned the same plant for any other use. The Fl values range from 0 to $100 \%$. Medicinal plants that are widely used by the local people for certain ailment have higher Fl values those that are less popular.

\section{Percentage of Respondent Knowledge of medicinal plants (PRK\%)}

PRK values are useful to determine most common medicinal plants in the study area. PRK values of medicinal plants were estimated using the formula: (number of people interviewed citing species/the total number of people interviewed) x100 (Friedman et al. 1986).

\section{Results and Discussion}

A total of 124 medicinal plants from in and around Lawachara national park for the treatment 53 ailment through 245 formularies was recorded. These species belong to 65 families. For each species scientific name, local name, family, parts used, ailments to be treated, application mode treatment and citation frequency are presented (Table 1). Most cited medicinal plants families in the study area are Rutaceae, Lamiaceae, Mimosaceae, Meliaceae, Combretaceae, and Asteraceae. Diversity in parts used for medicines was recorded in the study area. Leaf is the dominant parts used followed by Fruits, root and rhizome, whole plants, seeds, stem, bark, leaf and bark, petiole, bulb, peduncle, latex and flowers (Fig. 1). Dominant parts used leaf indicated that sustainable used of medicinal plants exist in the study area. In case roots and bark used may promote extinction process of species from nature. Part used fruits and seeds also created problem in natural regeneration of plants, if they do not collect properly. 
Knowledge of ethnomedical plants

Table 1. Ethno botanical data on medicinal plants and uses in the study area $(\mathrm{S}=\mathrm{Shrub}, \mathrm{C}=\mathrm{Climber}$, $\mathrm{H}=$ Herb, $\mathrm{T}=$ Tree)

\begin{tabular}{|c|c|c|c|c|c|c|}
\hline Scientific name, Voucher number & Local name & Family & Habit & Ailments & Part used & Treatment mode \\
\hline Abroma augusta (L.) L. f., Z-223 & Ulatkombol & Sterculiaceae & $\mathrm{s}$ & Impotence & Stem & Juice is taken \\
\hline Abrus precatorious L., Z-224 & Jostimodhu & Fabaceae & $\mathrm{c}$ & Cough & Stem & Juice is taken \\
\hline Achyranthes asperaL., Z-88 & Upathlenga & Amaranthaceae & $\mathrm{h}$ & Jaundice & Leaf & Juice is taken \\
\hline Acorus calamus L,. Z- 225 & Bach & Araceae & $\mathrm{h}$ & Appetizer & Rhizome & $\begin{array}{l}\text { Cooked rhizome } \\
\text { is taken }\end{array}$ \\
\hline Justicia adhatoda L.,Z-22 & Bashak & Acanthaceae & $\mathrm{h}$ & cough & Leaf & Juice is taken \\
\hline \multirow[t]{3}{*}{ Aegle marmelos (L.) Corr., Z-226 } & Bel & Rutaceae & $\mathrm{t}$ & Dysentery & $\begin{array}{l}\text { Green } \\
\text { fruits }\end{array}$ & $\begin{array}{l}\text { Raw fruits is } \\
\text { taken }\end{array}$ \\
\hline & & & & Diarrhoea & $\begin{array}{l}\text { Green } \\
\text { fruits }\end{array}$ & $\begin{array}{l}\text { Green fruit is } \\
\text { taken }\end{array}$ \\
\hline & & & & Constipation & Fruits & Pulp is taken \\
\hline Allium сера L., Z-227 & Peaj & Liliaceae & $\mathrm{h}$ & Flue & Bulb & Juice is taken \\
\hline \multirow[t]{2}{*}{ Allium sativumL., Z -228 } & Roshun & Liliaceae & $\mathrm{h}$ & Gastric & Bulb & Juice is taken \\
\hline & & & & Cold, cough & Bulb & Juice is taken \\
\hline \multirow[t]{2}{*}{ Alocasia cuculata L., Z- 142} & Bishkachu & Araceae & $\mathrm{h}$ & Body ache & Rhizome & $\begin{array}{l}\text { Cooked rhizome } \\
\text { is taken }\end{array}$ \\
\hline & & & & $\begin{array}{l}\text { Rheumatic } \\
\text { pain }\end{array}$ & Root & Juice is taken \\
\hline $\begin{array}{l}\text { Alocasia macrorrhizos (L.) G. Don, } \\
\text { Z-229 }\end{array}$ & Mankachu & Araceae & $\mathrm{h}$ & $\begin{array}{l}\text { Rheumatic } \\
\text { pain }\end{array}$ & Rhizome & $\begin{array}{l}\text { Cooked rhizome } \\
\text { is taken }\end{array}$ \\
\hline Aloe vera $($ L.) Burm. f. , Z- 230 & Alovera & Aloaceae & $\mathrm{h}$ & Impotence & Leaf & Juice is taken \\
\hline \multirow[t]{5}{*}{ Alstonia scholaris (L.) R. Br., Z-141 } & Chatim & Apocynaceae & $\mathrm{e} t$ & Ringworm & Latex & Latex is applied \\
\hline & & & & Pimple & Latex & Latex is applied \\
\hline & & & & Dysentery & Bark & Juice is taken \\
\hline & & & & Diarrhoea & Bark & Juice is taken \\
\hline & & & & Abscess & Latex & Latex is applied \\
\hline $\begin{array}{l}\text { Alternenthera sessilis (L.) R. Br. Ex } \\
\text { Roem. \& Schult., Z- } 12\end{array}$ & Helencha & $\begin{array}{l}\text { Amaranthace } \\
\text { ae }\end{array}$ & $\mathrm{h}$ & Pox & Leaf & Juice is taken \\
\hline Amaranthus tricolor L., Z-275 & Laoshak & $\begin{array}{l}\text { Amaranthace } \\
\text { ae }\end{array}$ & $\mathrm{c}$ & $\begin{array}{l}\text { Reduced } \\
\text { pressure }\end{array}$ & Leaf & $\begin{array}{l}\text { Cooked leaf } \\
\text { taken }\end{array}$ \\
\hline \multirow[t]{4}{*}{ Amaranthus spinosus L., Z-89 } & Kanta dugi & $\begin{array}{l}\text { Amaranthace } \\
\text { ae }\end{array}$ & $\mathrm{h}$ & $\begin{array}{l}\text { Urinary } \\
\text { problem }\end{array}$ & Stem & $\begin{array}{l}\text { Decoction is } \\
\text { taken }\end{array}$ \\
\hline & & & & $\begin{array}{l}\text { Rheumatic } \\
\text { pain }\end{array}$ & Root & Juice is taken \\
\hline & & & & Dropsy & Root & Juice is taken \\
\hline & & & & Diabetes & WP & $\begin{array}{l}\text { Cooked plant is } \\
\text { taken }\end{array}$ \\
\hline $\begin{array}{l}\text { Amorphophallus bulbifer (Roxb.) } \\
\text { Blume, Z-58 }\end{array}$ & Olkachu & Araceae & $\mathrm{h}$ & $\begin{array}{l}\text { Rheumatic } \\
\text { pain }\end{array}$ & Rhizome & $\begin{array}{l}\text { Cooked rhizome } \\
\text { is taken }\end{array}$ \\
\hline Andrographis paniculata (Burm. f.) & Kalomegh & Acanthaceae & $\mathrm{h}$ & Malaria & WP & Juice is taken \\
\hline \multirow{3}{*}{ Wall. ex Nees., Z- 31} & & & & Diabetes & WP & Juice is taken \\
\hline & & & & Dermatitis & Leaf & Paste is applied \\
\hline & & & & Anthlemintic & Leaf & Juice is taken \\
\hline Annona squamosa L., Z-231 & Anaros & Annonaceae & $\mathrm{h}$ & Anthlemintic & $\begin{array}{l}\text { Fruits, } \\
\text { root }\end{array}$ & Juice is taken \\
\hline $\begin{array}{l}\text { Breonia chinensis (Lamk.) Capuron, } \\
\text { Z-276 }\end{array}$ & Kadam & Rubiaceae & $\mathrm{t}$ & $\begin{array}{l}\text { Rheumatic } \\
\text { pain }\end{array}$ & Leaf & $\begin{array}{l}\text { Heated leaf is } \\
\text { applied }\end{array}$ \\
\hline $\begin{array}{l}\text { Aphanamixis polystachya(Wall.) R. } \\
\text { N. Parker, Z-277 }\end{array}$ & Roina & Meliaceae & $\mathrm{t}$ & Dermatitis & Leaf & Tablet is taken \\
\hline Aristolochia indica L., Z-278 & Ishwarmul & Orchidaceae & $\mathrm{c}$ & Dysentery & Root & Juice is taken \\
\hline Asparagus racemosus Willd., Z-279 & Shatamuli & Liliaceae & $\mathrm{c}$ & Impotence & Root & Juice is taken \\
\hline Averrhoa carambolaL., Z-132 & Kamranga & Averrhoaceae & $\mathrm{t}$ & Jaundice & Fruits & Fruit is taken \\
\hline
\end{tabular}


Contd.

\begin{tabular}{|c|c|c|c|c|c|c|}
\hline Scientific name, Voucher number & Local name & Family & Habit & Ailments & Part used & Treatment mode \\
\hline \multirow[t]{8}{*}{ Azadirachta indica A. Juss., Z-38 } & Neem & Meliaceae & $\mathrm{t}$ & Toothache & Leaf & $\begin{array}{l}\text { Decoction used } \\
\text { for Gargling }\end{array}$ \\
\hline & & & & Malaria & Leaf & Tablet is taken \\
\hline & & & & Gastric & leaf & Juice is taken \\
\hline & & & & Fever & Leaf & Juice is taken \\
\hline & & & & Diabetes & Leaf & Juice is taken \\
\hline & & & & Dermatitis & Leaf & Paste is applied \\
\hline & & & & Anthlemintic & Leaf & Tablet is taken \\
\hline & & & & Pox & Leaf & Paste is applied \\
\hline Baccaurea ramiflora Lour., Z- 117 & Bhubi & Juphorbiaceae & $\mathrm{t}$ & Appetizer & Fruits & Fruit is taken \\
\hline \multirow[t]{2}{*}{ Bambusa tulda Roxb., Z-171 } & Bamboo & Poaceae & s & Impotence & stem & $\begin{array}{l}\text { Cooked stem } \\
\text { taken }\end{array}$ \\
\hline & & & & Cut & Stem & $\begin{array}{l}\text { Powder is } \\
\text { applied }\end{array}$ \\
\hline Bauhinia acuminata L., Z- 248 & Shetkanson & $\begin{array}{l}\text { Ceasalpiniace } \\
\text { ae }\end{array}$ & $\mathrm{t}$ & $\begin{array}{l}\text { Tears of } \\
\text { Eye }\end{array}$ & Leaf & Juice is applied \\
\hline Blumea lacera (Burm. f.) DC, Z-70 & Shialmutra & Asteraceae & $\mathrm{h}$ & Diarrhoea & Leaf & $\begin{array}{l}\text { Fried leaf is } \\
\text { taken }\end{array}$ \\
\hline Bombax ceibaL., Z-107 & Shimul & Bombacaceae & $\mathrm{t}$ & Impotence & Root & Juice is taken \\
\hline \multirow{4}{*}{$\begin{array}{l}\text { Bulbophyllum lilacinum Ridl., Z- } \\
247\end{array}$} & Ishwarmul & Orchidaceae & $\mathrm{h}$ & Impotence & leaf & Juice is taken \\
\hline & & & & Dysentery, & leaf & Juice is taken \\
\hline & & & & Diabetes & leaf & Juice is taken \\
\hline & & & & Heart pain & leaf & Juice is taken \\
\hline $\begin{array}{l}\text { Bursera serrata Wall.ex colebr., Z- } \\
246\end{array}$ & Neur & Burseraceae & $\mathrm{t}$ & Appetizer & Fruits & Fruit is taken \\
\hline Cajanus cajan (L.) Millsp.,Z-245 & Orhor & Fabaceae & s & Jaundice & Leaf & Juice is taken \\
\hline \multirow{2}{*}{$\begin{array}{l}\text { Calotropis procera (Ait.) R. Br.,Z- } \\
04\end{array}$} & Akanda & Asclepiadaceae & s & Ringworm & Leaf & Paste is applied \\
\hline & & & & $\begin{array}{l}\text { Rheumatic } \\
\text { pain }\end{array}$ & Leaf & $\begin{array}{l}\text { Heated leaf is } \\
\text { applied }\end{array}$ \\
\hline Canabis sativa L., Z-249 & Gaza & Malvaceae & $\mathrm{s}$ & $\begin{array}{l}\text { Reduced } \\
\text { pressure }\end{array}$ & Leaf & Juice is taken \\
\hline \multirow[t]{3}{*}{ Carica papaya L., Z- 17} & Pepe & Caricaceae & $\mathrm{s}$ & $\begin{array}{l}\text { Stomach } \\
\text { ache }\end{array}$ & Fruits & $\begin{array}{l}\text { Cooked fruit is } \\
\text { taken }\end{array}$ \\
\hline & & & & Jaundice & Fruits & $\begin{array}{l}\text { Cooked fruit is } \\
\text { taken }\end{array}$ \\
\hline & & & & Gastric & Fruits & $\begin{array}{l}\text { Cooked fruit is } \\
\text { taken }\end{array}$ \\
\hline Careya arborea Roxb., Z-244 & Bidipata & Lecythidaceae & $\mathrm{t}$ & Dysentery & Leaf & Juice is taken \\
\hline Cassia alata L., Z-250 & Daudgash & Caesalpiniaceae & $\mathrm{s}$ & Ringworm & Leaf & Juice is applied \\
\hline $\begin{array}{l}\text { Cassinopsis ilicifolia (Hochst.) } \\
\text { Sleumer., Z- } 100\end{array}$ & Kantalebu & Rutaceae & s & Fever & Fruits & Juice is taken \\
\hline \multirow[t]{7}{*}{ Centella asiatica (L.) Urban, Z- 52} & Tunimakuni & Apiaceae & $\mathrm{h}$ & $\begin{array}{l}\text { Urinary } \\
\text { problem }\end{array}$ & WP & $\begin{array}{l}\text { Cooked plant is } \\
\text { taken }\end{array}$ \\
\hline & & & & Jaundice & $\mathrm{Wp}$ & Juice is taken \\
\hline & & & & Gastric & WP & $\begin{array}{l}\text { Cooked plant is } \\
\text { taken }\end{array}$ \\
\hline & & & & Dysentery & WP & Paste is taken \\
\hline & & & & Diarrhoea & WP & $\begin{array}{l}\text { Cooked plant is } \\
\text { taken }\end{array}$ \\
\hline & & & & $\begin{array}{l}\text { Cataract } \\
\text { eye }\end{array}$ & Leaf & Juice is applied \\
\hline & & & & Brain tonic & WP & Juice is taken \\
\hline $\begin{array}{l}\text { Ceriscoides campanulata (Roxb.) } \\
\text { Tirveng., Z-239 }\end{array}$ & Behlom & Rubiaceae & s & Jaundice & Fruits & $\begin{array}{l}\text { Cooked fruit is } \\
\text { taken }\end{array}$ \\
\hline
\end{tabular}


Contd.

\begin{tabular}{|c|c|c|c|c|c|c|}
\hline Scientific name, Voucher number & Local name & Family & Habit & Ailments & Part used & Treatment mode \\
\hline $\begin{array}{l}\text { Phyllanthus acidus (L.) Merr., Z- } \\
251\end{array}$ & Leboi & Euphorbiaceae & $\mathrm{t}$ & Fever & Fruits & Fruit is taken \\
\hline $\begin{array}{l}\text { Citrus aurantifolia (Christm. \& } \\
\text { Panzer) Swingle, Z- } 40\end{array}$ & Lebu & Rutaceae & $\mathrm{s}$ & Jaundice & Fruits & Juice is taken \\
\hline \multirow[t]{5}{*}{$\begin{array}{l}\text { Clerodendrum viscosum Pers., Z- } \\
238\end{array}$} & Bhait & Verbenaceae & $\mathrm{h}$ & $\begin{array}{l}\text { Stomach } \\
\text { ache }\end{array}$ & Leaf & Juice is taken \\
\hline & & & & Dysentery & Leaf & Juice is taken \\
\hline & & & & Cut & Leaf & Juice is applied \\
\hline & & & & Cough & Leaf & Juice is taken \\
\hline & & & & Anthlemintic & Leaf & Juice is taken \\
\hline Coccinia cordifolia Congn., Z- 13 & Telakucha & $\begin{array}{l}\text { Cucurbitacea } \\
\text { e }\end{array}$ & $\mathrm{c}$ & Diabetes & Leaf & $\begin{array}{l}\text { Cooked leaf } \\
\text { taken }\end{array}$ \\
\hline \multirow[t]{2}{*}{ Cocos nucifera L., Z-240 } & Narikel & Arecaceae & $\mathrm{t}$ & Jaundice & $\begin{array}{l}\text { Green } \\
\text { fruits }\end{array}$ & Water is taken \\
\hline & & & & Diarrhoea & $\begin{array}{l}\text { Green } \\
\text { fruits }\end{array}$ & Water is taken \\
\hline \multirow[t]{2}{*}{$\begin{array}{l}\text { Colocasia esculenta (L.) Schott., Z- } \\
08\end{array}$} & Kachu & Araceae & $\mathrm{h}$ & Iron tonic & WP & $\begin{array}{l}\text { Cooked plant is } \\
\text { taken }\end{array}$ \\
\hline & & & & Cut & Leaf & Juice is applied \\
\hline Crinum asiaticum Roxb.,Z-209 & Crinum & Liliaceae & $\mathrm{h}$ & Cow gastric & Fruits & Paste is taken \\
\hline $\begin{array}{l}\text { Curcuma zedoaria (Christm.) } \\
\text { Rosc., Z-281 }\end{array}$ & Shathi & $\begin{array}{l}\text { Zingiberac } \\
\text { eae }\end{array}$ & $\mathrm{h}$ & Diarrhoea & $\begin{array}{l}\text { Rhizo } \\
\text { me }\end{array}$ & $\begin{array}{l}\text { Powder is } \\
\text { taken }\end{array}$ \\
\hline Cuscuta reflexa Roxb.,Z-280 & Cuscuta & $\begin{array}{l}\text { Cuscutace } \\
\text { ae }\end{array}$ & $\mathrm{h}$ & Jaundice & Stem & Juice is taken \\
\hline \multirow[t]{2}{*}{$\begin{array}{l}\text { Cynodon dactylon (L.) Pers., Z- } \\
158\end{array}$} & Durba & Poaceae & $\mathrm{h}$ & Cut & WP & $\begin{array}{l}\text { Juice is } \\
\text { applied }\end{array}$ \\
\hline & & & & $\begin{array}{l}\text { Cut and } \\
\text { wound }\end{array}$ & Leaf & $\begin{array}{l}\text { Paste is } \\
\text { applied }\end{array}$ \\
\hline Dalbergia sissoo Roxb.,Z-274 & Sissue & Fabaceae & $\mathrm{t}$ & Jaundice & Leaf & Juice is taken \\
\hline Datura metel L.,Z-273 & Dutra & Solanaceae & $\mathrm{s}$ & Dermatitis & seed & $\begin{array}{l}\text { Paste is } \\
\text { applied }\end{array}$ \\
\hline Dillenia indica L., Z- 143 & Chalta & $\begin{array}{l}\text { Dilleniace } \\
\text { ae }\end{array}$ & $\mathrm{t}$ & $\begin{array}{l}\text { Reduced } \\
\text { pressure }\end{array}$ & Fruits & Fruit is taken \\
\hline Dillenia pentagyna Roxb., Z-242 & Harganja & $\begin{array}{l}\text { Dilleniace } \\
\text { ae }\end{array}$ & $\mathrm{t}$ & $\begin{array}{l}\text { Fractured } \\
\text { bone }\end{array}$ & $\begin{array}{l}\text { Leaf, } \\
\text { Bark }\end{array}$ & $\begin{array}{l}\text { Paste is } \\
\text { applied }\end{array}$ \\
\hline \multirow[t]{2}{*}{ Eclipta prostrata (L.) Mant, Z-11 } & Kesharaj & Asteraceae & $\mathrm{h}$ & Impotence & WP & Juice is taken \\
\hline & & & & Hair tonic & $\mathrm{Wp}$ & $\begin{array}{l}\text { Juice is } \\
\text { applied }\end{array}$ \\
\hline $\begin{array}{l}\text { Entada scandens auct. non Benth., } \\
\text { Z-241 }\end{array}$ & Gila & $\begin{array}{l}\text { Mimosace } \\
\text { ae }\end{array}$ & $\mathrm{c}$ & $\begin{array}{l}\text { Rheumatic } \\
\text { pain }\end{array}$ & Seed & Seed is taken \\
\hline Erythrina indica Lamk., Z-14 & Mandar & Fabaceae & $\mathrm{t}$ & Jaundice & Leaf, Bark & Juice is taken \\
\hline $\begin{array}{l}\text { Chromolaena odoratum(L.) King } \\
\text { and Robinson, Z- } 272\end{array}$ & Pisais & Asteraceae & $\mathrm{h}$ & Cut & Leaf & $\begin{array}{l}\text { Paste is } \\
\text { applied }\end{array}$ \\
\hline Paederia foetida L. Z-272 & padra pata & Rubiaceae & $\mathrm{c}$ & Diarrhoea & Leaf & $\begin{array}{l}\text { Cooked leaf } \\
\text { taken }\end{array}$ \\
\hline Ficus benghalensis L., Z- 77 & Bot & Moraceae & $\mathrm{t}$ & Diabetes & Fruits & $\begin{array}{l}\text { Cooked fruit } \\
\text { taken }\end{array}$ \\
\hline Ficus racemosa $\mathrm{L} ., \mathrm{Z}-271$ & Jogdumur & Moraceae & $\mathrm{t}$ & Diabetes & Fruits & $\begin{array}{l}\text { Cooked leaf } \\
\text { taken }\end{array}$ \\
\hline Cyperus rotundus L., Z-168 & Gandhavadlu & Cyperaceae & $\mathrm{c}$ & Diarrhoea & Leaf & $\begin{array}{l}\text { Cooked leaf } \\
\text { taken }\end{array}$ \\
\hline $\begin{array}{l}\text { Garcinia cawa Roxb. ex DC., Z- } \\
270\end{array}$ & Kao & Clusiaceae & $\mathrm{t}$ & Appetizer & Fruits & Fruit is taken \\
\hline $\begin{array}{l}\text { Garcinia xanthochymous Hook. f. } \\
\text { ex T. Anders, Z-269 }\end{array}$ & Dayphal & Clusiaceae & $\mathrm{t}$ & Appetizer & Fruits & Fruit is taken \\
\hline
\end{tabular}


Contd.

\begin{tabular}{|c|c|c|c|c|c|c|}
\hline Scientific name, Voucher number & Local name & Family & Habit & Ailments & Part used & Treatment mode \\
\hline \multirow[t]{9}{*}{$\begin{array}{l}\text { Glycosmis arborea (Roxb.) A. } \\
\text { DC., Z-20 }\end{array}$} & \multirow[t]{9}{*}{ Awapata } & \multirow[t]{9}{*}{ Rutaceae } & \multirow[t]{9}{*}{$\mathrm{s}$} & $\begin{array}{l}\text { Stomach } \\
\text { ache }\end{array}$ & Leaf & Juice is taken \\
\hline & & & & Jaundice & Leaf & Juice is taken \\
\hline & & & & Heart pain & Leaf & Juice is taken \\
\hline & & & & Head ache & Leaf & $\begin{array}{l}\text { Leaf paste is } \\
\text { applied }\end{array}$ \\
\hline & & & & Fever, & Leaf & Juice is taken \\
\hline & & & & Dysentery & Leaf & Juice is taken \\
\hline & & & & Cough & Leaf & Juice is taken \\
\hline & & & & Appetizer & Leaf & Juice is taken \\
\hline & & & & $\begin{array}{l}\text { Anthlemin } \\
\text { tic }\end{array}$ & Leaf & Juice is taken \\
\hline Hibiscus sabdariffa L., Z-60 & Amila & Malvaceae & $\mathrm{h}$ & Jaundice & Leaf & $\begin{array}{l}\text { Cooked leaf } \\
\text { is taken }\end{array}$ \\
\hline $\begin{array}{l}\text { Hygrophila spinosa T. Anders., Z- } \\
268\end{array}$ & Talmakhna & $\begin{array}{l}\text { Acanthace } \\
\text { ae }\end{array}$ & $\mathrm{h}$ & $\begin{array}{l}\text { Eye } \\
\text { complain }\end{array}$ & Seed & $\begin{array}{l}\text { Juice is } \\
\text { applied }\end{array}$ \\
\hline $\begin{array}{l}\text { Hydnocarpus kurzii } \\
\text { (King)Warb.,Z-87 }\end{array}$ & Chalmugra & $\begin{array}{l}\text { Archariace } \\
\text { ae }\end{array}$ & $\mathrm{t}$ & Leprosy & Fruits & Oil is applied \\
\hline \multirow[t]{3}{*}{ Hyptis suaveolens (L.) Poit., Z-267 } & \multirow[t]{3}{*}{ Tokma } & \multirow[t]{3}{*}{ Lamiaceae } & $\mathrm{h}$ & $\begin{array}{l}\text { Dysentery } \\
\text { Reduced } \\
\text { pressure }\end{array}$ & $\begin{array}{l}\text { Root } \\
\text { seed }\end{array}$ & $\begin{array}{l}\text { Juice is taken } \\
\text { Seed is taken }\end{array}$ \\
\hline & & & $\mathrm{h}$ & Dysentery & Seed & Juice is taken \\
\hline & & & $\mathrm{h}$ & $\begin{array}{l}\text { Constipati } \\
\text { on }\end{array}$ & seed & Seed is taken \\
\hline $\begin{array}{l}\text { Ipomoea fistulosa Mart. ex Choisy, } \\
\text { Z-203 }\end{array}$ & Khulum & $\begin{array}{l}\text { Convolvul } \\
\text { aceae }\end{array}$ & $\mathrm{h}$ & Cut & Latex & $\begin{array}{l}\text { Latex is } \\
\text { applied }\end{array}$ \\
\hline Ipomoea mauritiana Jacq., Z-265 & Bhuikumra & $\begin{array}{l}\text { Convolvul } \\
\text { aceae }\end{array}$ & $\mathrm{c}$ & $\begin{array}{l}\text { Miscarriag } \\
\mathrm{e}\end{array}$ & Root & Juice is taken \\
\hline Jatropha curcas L., Z-266 & Keke & $\begin{array}{l}\text { Euphorbia } \\
\text { ceae }\end{array}$ & $\mathrm{s}$ & Toothache & Leaf & $\begin{array}{l}\text { Juice is } \\
\text { applied }\end{array}$ \\
\hline \multirow[t]{3}{*}{ Kalanchoe pinnata (Lamk.), Z-46 } & \multirow[t]{3}{*}{ Pathorkuch } & \multirow[t]{3}{*}{ Crassulaceae } & \multirow[t]{3}{*}{ h } & $\begin{array}{l}\text { Urinary } \\
\text { problem }\end{array}$ & Leaf & Juice is taken \\
\hline & & & & Dysentery & Leaf & Juice is taken \\
\hline & & & & Cough & Leaf & Juice is taken \\
\hline \multirow[t]{3}{*}{$\begin{array}{l}\text { Leucas lavandulaefolia Smith., Z- } \\
28\end{array}$} & \multirow[t]{3}{*}{$\begin{array}{l}\text { Dandakala } \\
\text { sh }\end{array}$} & \multirow[t]{3}{*}{ Lamiaceae } & \multirow[t]{3}{*}{$\mathrm{h}$} & $\begin{array}{l}\text { Stomach } \\
\text { ache }\end{array}$ & Leaf & Juice is taken \\
\hline & & & & Diarrhoea & Leaf & $\begin{array}{l}\text { Fried leaf is } \\
\text { taken }\end{array}$ \\
\hline & & & & Cough & Leaf & $\begin{array}{l}\text { Fried leaf is } \\
\text { taken }\end{array}$ \\
\hline \multirow[t]{4}{*}{$\begin{array}{l}\text { Litsea glutinosa (Lour.) Robinson, } \\
\text { Z- } 94\end{array}$} & \multirow[t]{4}{*}{ Chengpisla } & \multirow[t]{4}{*}{ Lauraceae } & \multirow[t]{4}{*}{$\mathrm{t}$} & $\begin{array}{l}\text { Stomach } \\
\text { ache }\end{array}$ & $\begin{array}{l}\text { Leaf, } \\
\text { Bark }\end{array}$ & Juice is taken \\
\hline & & & & Impotence & $\begin{array}{l}\text { Leaf, } \\
\text { Bark }\end{array}$ & Juice is taken \\
\hline & & & & Dysentery & $\begin{array}{l}\text { Leaf, } \\
\text { Bark }\end{array}$ & Juice is taken \\
\hline & & & & $\begin{array}{l}\text { Constipati } \\
\text { on }\end{array}$ & $\begin{array}{l}\text { Leaf, } \\
\text { Bark }\end{array}$ & Juice is taken \\
\hline \multirow[t]{5}{*}{ Lawsonia inermis L., Z- 35} & \multirow[t]{5}{*}{ Mehedi } & \multirow[t]{5}{*}{ Lythraceae } & \multirow[t]{5}{*}{$\mathrm{s}$} & Impotence & leaf & Juice is taken \\
\hline & & & & Impotence & leaf & Juice is taken \\
\hline & & & & Hair tonic & Leaf & $\begin{array}{l}\text { Paste is } \\
\text { applied }\end{array}$ \\
\hline & & & & Gastric & Leaf & Juice is taken \\
\hline & & & & $\begin{array}{l}\text { Birth } \\
\text { control }\end{array}$ & Leaf & Juice is taken \\
\hline
\end{tabular}


Contd.

\begin{tabular}{|c|c|c|c|c|c|c|}
\hline Scientific name, Voucher number & Local name & Family & Habit & Ailments & Part used & Treatment mode \\
\hline \multirow[t]{2}{*}{ Mangifera indica L., Z-243 } & \multirow[t]{2}{*}{ Aam } & \multirow[t]{2}{*}{$\begin{array}{l}\text { Anacardia } \\
\text { ceae }\end{array}$} & \multirow[t]{2}{*}{$\mathrm{t}$} & Toothache & Leaf & $\begin{array}{l}\text { Juice is } \\
\text { applied }\end{array}$ \\
\hline & & & & Dysentery & Leaf & Paste is taken \\
\hline $\begin{array}{l}\text { Melia sempervirens (L.) Sw., Z- } \\
34\end{array}$ & Bela & Meliaceae & $\mathrm{t}$ & Dermatitis & Leaf & $\begin{array}{l}\text { Paste is } \\
\text { applied }\end{array}$ \\
\hline Mentha arvensis L., Z-264 & Pudina & Lamiaceae & $\mathrm{h}$ & $\begin{array}{l}\text { Stomach } \\
\text { ache }\end{array}$ & Leaf & Juice is taken \\
\hline \multirow{2}{*}{$\begin{array}{l}\text { Mikania cordata (Burm. f.) B.L. } \\
\text { Rob., Z-261 }\end{array}$} & \multirow[t]{2}{*}{ Refujeelata } & \multirow[t]{2}{*}{ Asteraceae } & \multirow[t]{2}{*}{$\mathrm{c}$} & Dysentery & Leaf & Juice is taken \\
\hline & & & & Cut & Leaf & $\begin{array}{l}\text { Juice is } \\
\text { applied }\end{array}$ \\
\hline \multirow[t]{9}{*}{ Mimosa pudica L., Z-15 } & \multirow[t]{9}{*}{ Chaitamara } & \multirow[t]{9}{*}{ Mimosaceae } & \multirow[t]{9}{*}{$\mathrm{h}$} & Waist pain & root & Juice is taken \\
\hline & & & & Toothache & Root & $\begin{array}{l}\text { Juice is } \\
\text { applied }\end{array}$ \\
\hline & & & & Measles & Stem & $\begin{array}{l}\text { Juice is } \\
\text { applied }\end{array}$ \\
\hline & & & & Malaria & Root & Juice is taken \\
\hline & & & & Jaundice & Leaf & Juice is taken \\
\hline & & & & Fever & Leaf & Juice is taken \\
\hline & & & & $\begin{array}{l}\text { Anthlemin } \\
\text { tic }\end{array}$ & Leaf & Juice is taken \\
\hline & & & & Abscess & Root & $\begin{array}{l}\text { Paste is } \\
\text { applied }\end{array}$ \\
\hline & & & & $\begin{array}{l}\text { During } \\
\text { birth }\end{array}$ & Root & $\begin{array}{l}\text { Paste is } \\
\text { applied }\end{array}$ \\
\hline \multirow[t]{3}{*}{ Acacia intisia (L.) Willd., Z-262 } & \multirow[t]{3}{*}{$\begin{array}{l}\text { Sadachait } \\
\text { amara }\end{array}$} & \multirow[t]{3}{*}{$\begin{array}{l}\text { Mimosace } \\
\text { ae }\end{array}$} & \multirow[t]{3}{*}{$\mathrm{h}$} & Snakebite & Root & $\begin{array}{l}\text { Juice is } \\
\text { applied }\end{array}$ \\
\hline & & & & Impotence & root & Juice is taken \\
\hline & & & & Allergy & Root & Juice is taken \\
\hline \multirow[t]{3}{*}{ Moringa oleifera Lamk.,Z-252 } & \multirow[t]{3}{*}{ Sajna } & \multirow[t]{3}{*}{$\begin{array}{l}\text { Moringace } \\
\text { ae }\end{array}$} & \multirow[t]{3}{*}{$\mathrm{t}$} & $\begin{array}{l}\text { Stomach } \\
\text { ache }\end{array}$ & Fruits & $\begin{array}{l}\text { Cooked fruit is } \\
\text { taken }\end{array}$ \\
\hline & & & & $\begin{array}{l}\text { Rheumatic } \\
\text { pain }\end{array}$ & $\begin{array}{l}\text { Leaf, } \\
\text { Bark }\end{array}$ & $\begin{array}{l}\text { Cooked leaf is } \\
\text { taken }\end{array}$ \\
\hline & & & & Diarrhoea & Leaf & Juice is taken \\
\hline \multirow[t]{2}{*}{$\begin{array}{l}\text { Murraya paniculata (L.) Jack., Z- } \\
25\end{array}$} & \multirow[t]{2}{*}{ Kamini } & \multirow[t]{2}{*}{ Rutaceae } & \multirow[t]{2}{*}{$\mathrm{t}$} & Toothache & Leaf & $\begin{array}{l}\text { Juice is } \\
\text { applied }\end{array}$ \\
\hline & & & & Toothache & Leaf & $\begin{array}{l}\text { Juice is } \\
\text { applied }\end{array}$ \\
\hline \multirow[t]{2}{*}{$\begin{array}{l}\text { Murrya koenigii (L.) Spreng., Z- } \\
254\end{array}$} & \multirow[t]{2}{*}{ Norshing } & \multirow[t]{2}{*}{ Rutaceae } & \multirow[t]{2}{*}{$\mathrm{s}$} & Diarrhoea, & Leaf & $\begin{array}{l}\text { Cooked leaf is } \\
\text { taken }\end{array}$ \\
\hline & & & & Cough & Leaf & $\begin{array}{l}\text { Cooked leaf is } \\
\text { taken }\end{array}$ \\
\hline \multirow[t]{3}{*}{ Musa paradisiaca L, Z-253. } & Kola & Musaceae & $\mathrm{h}$ & Dysentery & Fruits & Fruit is taken \\
\hline & & & & Cut & $\begin{array}{l}\text { Pedun } \\
\text { cle }\end{array}$ & $\begin{array}{l}\text { Juice is } \\
\text { applied }\end{array}$ \\
\hline & & & & $\begin{array}{l}\text { Constipati } \\
\text { on }\end{array}$ & $\begin{array}{l}\text { Pedun } \\
\text { cle }\end{array}$ & $\begin{array}{l}\text { Cooked } \\
\text { peduncle is } \\
\text { taken }\end{array}$ \\
\hline $\begin{array}{l}\text { Nicotiana plumbaginifolia Viv., } \\
\text { Z-255 }\end{array}$ & Tamak & Solanaceae & $\mathrm{h}$ & Cut & Leaf & $\begin{array}{l}\text { Juice is } \\
\text { applied }\end{array}$ \\
\hline Nigella sativa L., Z-256 & Kalojira & $\begin{array}{l}\text { Ranuncula } \\
\text { ceae }\end{array}$ & $\mathrm{h}$ & Impotence & seed & Oil is taken \\
\hline & & & & Diabetes & seed & $\begin{array}{l}\text { Water extract } \\
\text { is taken }\end{array}$ \\
\hline $\begin{array}{l}\text { Nyctanthes arbor-tristis L., Z- } \\
257\end{array}$ & Shephaliful & $\begin{array}{l}\text { Nyctagina } \\
\text { ceae }\end{array}$ & $\mathrm{s}$ & Fever & Leaf & Juice is taken \\
\hline
\end{tabular}


Contd.

\begin{tabular}{|c|c|c|c|c|c|c|}
\hline Scientific name, Voucher number & Local name & Family & Habit & Ailments & Part used & Treatment mode \\
\hline & & & & Anthlemintic & Leaf & Juice is taken \\
\hline \multirow[t]{4}{*}{ Ocimum americanum L. Z-237 } & Rossetpata & Lamiaceae & $\mathrm{h}$ & $\begin{array}{l}\text { Stomach } \\
\text { ache }\end{array}$ & Leaf & $\begin{array}{l}\text { Cooked leaf is } \\
\text { taken }\end{array}$ \\
\hline & & & & Gastric & Leaf & $\begin{array}{l}\text { Cooked leaf is } \\
\text { taken }\end{array}$ \\
\hline & & & & Diarrhoea & Leaf & Juice is taken \\
\hline & & & & Cough & Leaf & Juice is taken \\
\hline \multirow[t]{3}{*}{ Ocimum basilicumL., Z-02 } & Kalotulsi & Lamiaceae & $\mathrm{h}$ & $\begin{array}{l}\text { Reduced } \\
\text { pressure }\end{array}$ & Leaf & $\begin{array}{l}\text { Paste is } \\
\text { applied }\end{array}$ \\
\hline & & & & fever & leaf & Juice is taken \\
\hline & & & & Cough & Leaf & Juice is taken \\
\hline \multirow[t]{3}{*}{ Ocimum sanctum L., Z-153 } & Sada tulsi & Lamiaceae & $\mathrm{h}$ & $\begin{array}{l}\text { Reduced } \\
\text { pressure }\end{array}$ & Leaf & $\begin{array}{l}\text { Paste is } \\
\text { applied }\end{array}$ \\
\hline & & & & Cough & Leaf & Juice is taken \\
\hline & & & & cough & Leaf & Juice is taken \\
\hline $\begin{array}{l}\text { Oroxylum indicum (L.) Kurz., Z- } \\
110\end{array}$ & Kanaidingi & $\begin{array}{l}\text { Bignoniac } \\
\text { eae }\end{array}$ & $\mathrm{t}$ & Jaundice & Bark & $\begin{array}{l}\text { Juice is } \\
\text { applied }\end{array}$ \\
\hline Oryza sativa L., Z-236 & Dhan & Poaceae & $\mathrm{H}$ & Diarrhoea & Seed & $\begin{array}{l}\text { Powder is } \\
\text { taken }\end{array}$ \\
\hline \multirow[t]{2}{*}{ Oxalis corniculata $\mathrm{L} ., \mathrm{Z}-23$} & Zinzil & Oxalidaceae & $\mathrm{h}$ & $\begin{array}{l}\text { Stomach } \\
\text { ache }\end{array}$ & Leaf & Juice is taken \\
\hline & & & & Appetizer & Leaf & Juice is taken \\
\hline Pandanus foetidus Roxb., Z--235 & Keya & $\begin{array}{l}\text { Pandanace } \\
\text { ae }\end{array}$ & $\mathrm{H}$ & Cough & $\begin{array}{l}\text { Rhizo } \\
\text { me }\end{array}$ & Juice is taken \\
\hline \multirow[t]{2}{*}{ Phyllanthus emblicaL., Z-44 } & Aola & $\begin{array}{l}\text { Euphorbia } \\
\text { ceae }\end{array}$ & $\mathrm{t}$ & Impotence & Fruits & Fruit is taken \\
\hline & & & & Appetizer & Fruits & Fruit is taken \\
\hline \multirow[t]{2}{*}{ Piper betel L., Z- 187} & Pan & Piperaceae & $\mathrm{c}$ & Cut & Leaf & $\begin{array}{l}\text { Juice is } \\
\text { applied }\end{array}$ \\
\hline & & & & cut & Leaf & $\begin{array}{l}\text { Juice is } \\
\text { applied }\end{array}$ \\
\hline Plumbago zeylanica L., Z-261 & Chita & $\begin{array}{l}\text { Plumbagin } \\
\text { aceae }\end{array}$ & $\mathrm{h}$ & Dermatitis & Root & $\begin{array}{l}\text { Juice is } \\
\text { applied }\end{array}$ \\
\hline $\begin{array}{l}\text { Cnesmone javanica Blume, Z- } \\
260\end{array}$ & Chutra & $\begin{array}{l}\text { Euphorbia } \\
\text { ceae }\end{array}$ & $\mathrm{h}$ & Dermatitis & Leaf & $\begin{array}{l}\text { Juice is } \\
\text { applied }\end{array}$ \\
\hline \multirow[t]{2}{*}{$\begin{array}{l}\text { Persicaria hydropiper (L.), } \\
\text { Spach Z-138 }\end{array}$} & Bishkatali & $\begin{array}{l}\text { Polygonac } \\
\text { eae }\end{array}$ & $\mathrm{h}$ & Dermatitis & Leaf & $\begin{array}{l}\text { Juice is } \\
\text { applied }\end{array}$ \\
\hline & & & & Jaundice & Leaf & Juice is taken \\
\hline \multirow[t]{2}{*}{$\begin{array}{l}\text { Chylocalyx perfoliatus (L.) } \\
\text { Hassk. ex Miq.L., Z- } 48\end{array}$} & $\begin{array}{l}\text { Kantaamr } \\
\text { ai }\end{array}$ & $\begin{array}{l}\text { Polygonac } \\
\text { eae }\end{array}$ & $\mathrm{h}$ & Diabetes & Leaf & Paste is taken \\
\hline & & & & Cough & Leaf & $\begin{array}{l}\text { Fried leaf is } \\
\text { taken }\end{array}$ \\
\hline \multirow[t]{3}{*}{ Psidium guajavaL., Z- 43} & Peara & Myrtaceae & $\mathrm{t}$ & Toothache & Leaf & $\begin{array}{l}\text { Decoction is } \\
\text { used for } \\
\text { gargling }\end{array}$ \\
\hline & & & & Dysentery & Leaf & Juice is taken \\
\hline & & & & Diarrhoea & Leaf & Juice is taken \\
\hline \multirow[t]{2}{*}{ Ricinus communis L., Z-258 } & Verenda & $\begin{array}{l}\text { Euphorbia } \\
\text { ceae }\end{array}$ & $\mathrm{s}$ & $\begin{array}{l}\text { Constipati } \\
\text { on }\end{array}$ & Seed & Oil is taken \\
\hline & & & & Vomiting & Bark & $\begin{array}{l}\text { Juice is } \\
\text { applied }\end{array}$ \\
\hline Saccharum officinarum L., Z-234 & Akh & Poaceae & $\mathrm{h}$ & Jaundice & Stem & Juice is taken \\
\hline \multirow[t]{2}{*}{ Scoparia dulcis L., Z-16 } & Chinipata & $\begin{array}{l}\text { Scrophular } \\
\text { iaceae }\end{array}$ & $\mathrm{h}$ & $\begin{array}{l}\text { Eye } \\
\text { complain }\end{array}$ & Leaf & $\begin{array}{l}\text { Juice is } \\
\text { applied }\end{array}$ \\
\hline & & & & $\begin{array}{l}\text { Diarrhoea } \\
\text { Diabetes }\end{array}$ & $\begin{array}{l}\text { Leaf } \\
\text { Leaf }\end{array}$ & $\begin{array}{l}\text { Juice is taken } \\
\text { Juice is taken }\end{array}$ \\
\hline
\end{tabular}


Contd.

\begin{tabular}{|c|c|c|c|c|c|c|}
\hline Scientific name, Voucher number & Local name & Family & Habit & Ailments & Part used & Treatment mode \\
\hline Sesamum indicum L. Z-259 & Til & Pedaliaceae & $\mathrm{h}$ & Eczema & Seed & Oil is applied \\
\hline Smilax macrophylla Roxb., Z 233 & Kumarilata & $\begin{array}{l}\text { Smilacace } \\
\text { ae }\end{array}$ & $\mathrm{c}$ & Impotence & Leaf & $\begin{array}{l}\text { Leaf is taken } \\
\text { internally }\end{array}$ \\
\hline $\begin{array}{l}\text { Solanum violaceum Ortega, Z- } \\
129\end{array}$ & Boroibegun & Solanaceae & $\mathrm{s}$ & $\begin{array}{l}\text { Stomach } \\
\text { ache }\end{array}$ & Fruits & Paste is taken \\
\hline \multirow[t]{2}{*}{ Spilanthes acmella (L). L., Z- 19} & Piperman & Asteraceae & $\mathrm{h}$ & Toothache & Flowe & Juice is \\
\hline & & & & $\begin{array}{l}\text { Ear and } \\
\text { mouth rot }\end{array}$ & $\begin{array}{l}\text { rs } \\
\text { Flowe } \\
\text { rs }\end{array}$ & $\begin{array}{l}\text { applied } \\
\text { Juice is } \\
\text { applied }\end{array}$ \\
\hline \multirow[t]{6}{*}{$\begin{array}{l}\text { Sterculia villosa Roxb. ex Smith, } \\
\text { Z- } 125\end{array}$} & Udal & $\begin{array}{l}\text { Sterculiace } \\
\text { ae }\end{array}$ & $\mathrm{t}$ & Jaundice & Petiole & Juice is taken \\
\hline & & & & impotence & Petiole & Juice is taken \\
\hline & & & & Gastric & Petiole & Juice is taken \\
\hline & & & & Dysentery & Petiole & Juice is taken \\
\hline & & & & Diabetes & Petiole & Juice is taken \\
\hline & & & & $\begin{array}{l}\text { Constipati } \\
\text { on }\end{array}$ & Petiole & Juice is taken \\
\hline Streblus asper Lour., Z-84 & Sheora & Moraceae & $\mathrm{t}$ & Dysentery & Leaf & Juice is taken \\
\hline Swietenia mahagoni Jacq., Z-42 & Mehogoni & Meliaceae & $\mathrm{t}$ & Diabetes & Seed & Juice is taken \\
\hline $\begin{array}{l}\text { Syzygium cumini (L.) Skeels, Z- } \\
39\end{array}$ & Jam & Myrtaceae & $\mathrm{t}$ & Toothache & Leaf & $\begin{array}{l}\text { Juice is } \\
\text { applied }\end{array}$ \\
\hline \multirow[t]{2}{*}{ Tagetes erectaL., Z- 79} & Gada & Asteraceae & $\mathrm{h}$ & $\begin{array}{l}\text { Diabetes } \\
\text { Fire injury }\end{array}$ & $\begin{array}{l}\text { Seed } \\
\text { leaf }\end{array}$ & $\begin{array}{l}\text { Seed is taken } \\
\text { juice is } \\
\text { applied }\end{array}$ \\
\hline & & & & Cut & Leaf & $\begin{array}{l}\text { Juice is } \\
\text { applied }\end{array}$ \\
\hline \multirow[t]{4}{*}{ Tamarindus indica Willd., Z- 116} & Tentul & $\begin{array}{l}\text { Caesalpini } \\
\text { aceae }\end{array}$ & $\mathrm{t}$ & Sensitize & Fruits & Fruit is taken \\
\hline & & & & $\begin{array}{l}\text { Reduced } \\
\text { pressure }\end{array}$ & Fruits & Fruit is taken \\
\hline & & & & Diarrhoea & Fruits & Fruit is taken \\
\hline & & & & Appetizer & Fruits & Fruit is taken \\
\hline \multirow[t]{3}{*}{$\begin{array}{l}\text { Terminalia arjuna (Roxb. ex } \\
\text { DC.) Wight \& Arn., Z- } 130\end{array}$} & Arjun & $\begin{array}{l}\text { Combretac } \\
\text { eae }\end{array}$ & $\mathrm{t}$ & Heart pain & Bark & Juice is taken \\
\hline & & & & Gastric & Bark & Juice is taken \\
\hline & & & & Dysentery & Bark & Juice is taken \\
\hline \multirow[t]{4}{*}{$\begin{array}{l}\text { Terminalia bellirica (Gaertn.) } \\
\text { Roxb., Z- } 108\end{array}$} & Bohera & $\begin{array}{l}\text { Combretac } \\
\text { eae }\end{array}$ & $\mathrm{t}$ & $\begin{array}{l}\text { Stomach } \\
\text { ache }\end{array}$ & Fruits & Fruit is taken \\
\hline & & & & Impotence & Fruits & Fruit is taken \\
\hline & & & & Dysentery & Fruits & Fruit is taken \\
\hline & & & & Appetizer & Fruits & Fruit is taken \\
\hline \multirow[t]{4}{*}{ Terminalia chebula Retz., Z- 55} & Horitaki & $\begin{array}{l}\text { Combretac } \\
\text { eae }\end{array}$ & $\mathrm{t}$ & $\begin{array}{l}\text { Stomach } \\
\text { ache }\end{array}$ & Fruits & Fruit is taken \\
\hline & & & & Jaundice & Bark & $\begin{array}{l}\text { Decoction is } \\
\text { taken }\end{array}$ \\
\hline & & & & Impotence & Fruits & Fruit is taken \\
\hline & & & & Appetizer & Fruits & Fruit is taken \\
\hline $\begin{array}{l}\text { Tinospora crispa (L.) Hook. f. \& } \\
\text { Thoms, Z-232 }\end{array}$ & Gulancha & $\begin{array}{l}\text { Menisper } \\
\text { maceae }\end{array}$ & $\mathrm{c}$ & $\begin{array}{l}\text { Anthlemin } \\
\text { tic }\end{array}$ & Stem & Juice is taken \\
\hline Vitex negundo L., Z- 131 & Nishinda & Verbenaceae & $\mathrm{s}$ & cough & Leaf & Juice is taken \\
\hline $\begin{array}{l}\text { Vitis quadrangualris Wall. ex } \\
\text { Wight \& Arn., Z- } 136\end{array}$ & Harjora & Vitaceae & $\mathrm{c}$ & $\begin{array}{l}\text { Fractured } \\
\text { bone }\end{array}$ & Stem & $\begin{array}{l}\text { Paste is } \\
\text { applied }\end{array}$ \\
\hline Zingiber officinale Rosc., Z-114 & Ada & $\begin{array}{l}\text { Zingiberac } \\
\text { eae }\end{array}$ & $\mathrm{h}$ & Neck pain & $\begin{array}{l}\text { Rhizo } \\
\text { me }\end{array}$ & $\begin{array}{l}\text { Decoction is } \\
\text { taken }\end{array}$ \\
\hline $\begin{array}{l}\text { Ziziphus mauritiana Lamk., Z- } \\
05\end{array}$ & Boroi & $\begin{array}{l}\text { Rhamnace } \\
\text { ae }\end{array}$ & $\mathrm{t}$ & Wound & Leaf & $\begin{array}{l}\text { Boiled water } \\
\text { applied }\end{array}$ \\
\hline
\end{tabular}


Of 124 species documented in the study area, herbs have been represented by $43 \%$, trees by $31 \%$, shrubs by $15 \%$ and climbers by $11 \%$ of total species (Fig. 2). The result reflected that herbs are the most dominant life form among the medicinal plants in the study area. In order to maintain medicinal diversity in nature, herbaceous plants can be cultivated easily as a major source of crude drugs because of short life cycle. Usually people take medicines in different ways including oral application, external application and adjunct therapy. Oral application is the main mode of treatment in the study area and followed by external application (Fig. 3).

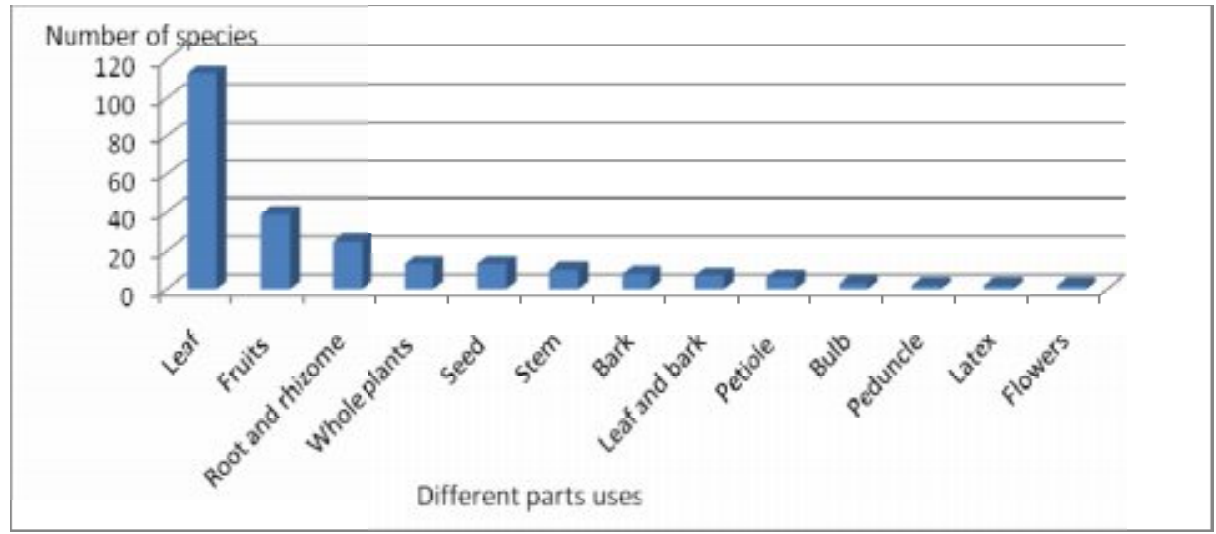

Fig. 1. Diversity in parts used of medicinal plants.

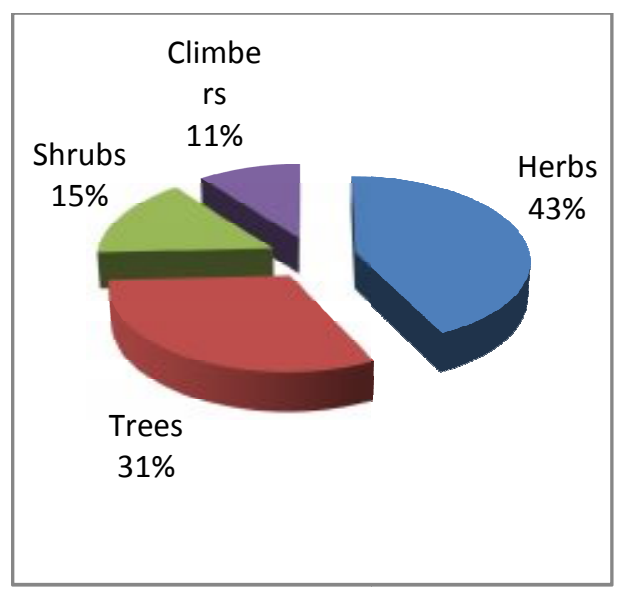

Fig. 2. Different life forms of species.
Used internally Used externally

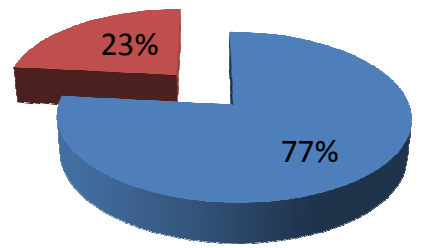

Fig. 3. Application modes of medicines. 


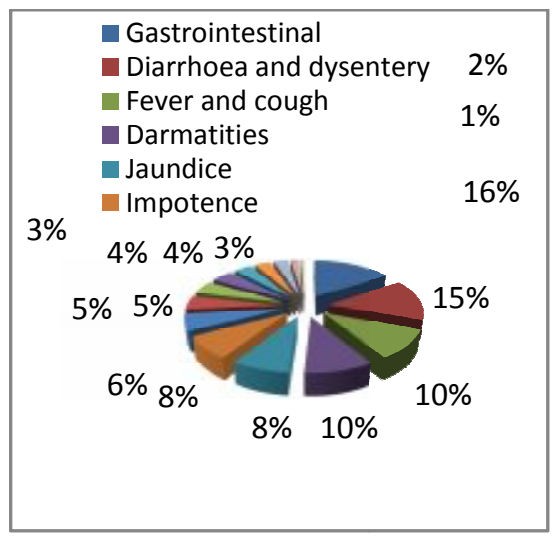

Fig. 4. Fomularies.

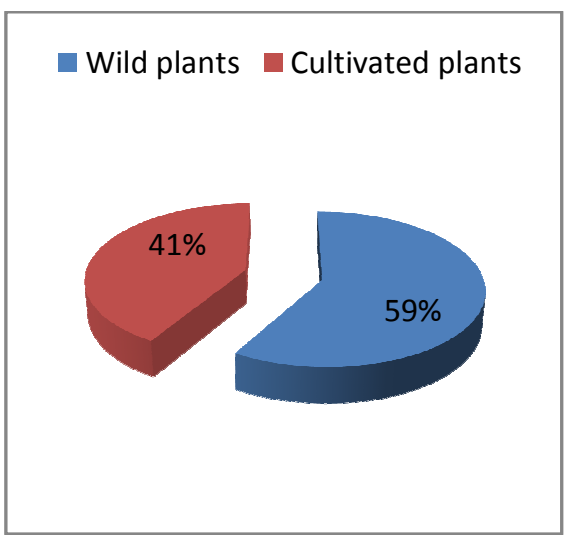

Fig. 5. Sources of medicinal plants.

Number of formularies for major ailment group showed variation. Maximum formularies were found in the six ailment groups including gastrointestinal complain, diarrhea and dysentery, fever and cough, dermatitis, jaundice and impotence (Fig. 4). It is proved that such complains are very common in the study area. According to the local people most plants $(59 \%)$ were harvested from the wild vegetation and minimum (41\%) harvested from cultivated plants (Fig.5). Cultivation of medicinal plants is still in low because of availability of wild vegetation in national park. It is observed in the field that harvesting of medicinal plants from the wild is not sustainable. Local people are not aware of sustainable use of medicinal plants. They like to earn some cash money for their livelihood by selling illegal plant parts from the wild. As far literature there is no appropriate policy and guide line for medicinal plants harvesting from the wild in Bangladesh. Currently some cultivation program of medicinal plants has been started here in Bangladesh. But herbal industries are yet depending on foreign supply for raw drug materials (Personal communication).

Table 2. Consensus of agreement in the uses of medicinal plants among the informants.

\begin{tabular}{lccc}
\hline Major ailments group & Nur & Nt & Fic \\
\hline Cut and wound & 188 & 15 & 0.93 \\
Jaundice & 196 & 21 & 0.90 \\
Respiratory related (malaria, fever and cough) & 235 & 26 & 0.90 \\
Diabetes & 90 & 12 & 0.88 \\
Diarrhoea and dysentery & 258 & 36 & 0.87 \\
Anthelmintic & 50 & 8 & 0.86 \\
Gastrointestinal complain & 263 & 39 & 0.86 \\
Impotence & 118 & 18 & 0.86 \\
Dermatitis & 155 & 24 & 0.85 \\
High blood pressure & 62 & 12 & 0.82 \\
Urinary disorder & 32 & 7 & 0.81 \\
Rheumatic pain & 49 & 11 & 0.79 \\
Toothache & 34 & 11 & 0.70 \\
Eye complain & 9 & 4 & 0.63 \\
\hline
\end{tabular}


Based on the information obtained from the informants, the recorded ailments were grouped into 14 categories (Table 2). The results could be useful in prioritizing medicinal plants for further scientific validation of plant products, as pharmacologically effective remedies with higher Fic values. Fic values range from 0.00 to 1.00 . High values are obtained when only one or few plants species are reported to be used by a high proportion of informants to treat a particular ailment, whereas low Fic values indicate that the informants disagree over which plants to use. Higher Fic values can thus be used to pinpoint particularly interesting species for the search of bioactive compounds. In our analysis Fic values for major ailment categories showed variation (Table 2). The average Fic value for all ailment categories obtained was0.78. Such value indicated that maximum people in the study area were well informed about the medicinal knowledge of plants and also showed agreement on the use of medicinal plants for such categories of ailments. Top Fic values found in case of ailment cut and wound. The most cited species used to treat such ailment are Mikania cordata, Chromolaena odoratum and Cynodon dactylon. Among them Cynodon dactylon and Chromolaena odoratum showed maximum Fl values.These species can be used for further phytochemical analysis to find active compounds for treatment of cut and wound. Jaundice and respiratory related (malaria, fever, cold and cough) treatment scored second highest Fic values. Most cited species to treat such categories of ailmentwere Ceriscoides campanulata, Oroxylum indicum, Cuscuta reflexa, Averrhoa carambola, Citrus aurantifolia, Cajanus cajan, Ocimum sanctum, Andrographis paniculata, Ocimum basilicum and Justicia adhatoda. Such species can be used for further phytochemical analysis to find active compounds for the treatment of Jaundice and respiratory related ailments.

Fidelity level is useful for identifying the key informants most preferred species used for treating certain ailments. The medicinal plants that are widely used by the local people have higher FL values than those that are less used. On the other hand, medicinal plants that are known as remedies of a single aliment have $100 \%$ fidelity level than those that are used as remedies for more than one type of aliment. In our analysis Fl values showed variations. Among the most cited species 9 scored $\mathrm{Fl}$ values of $100 \%$. The maximum $\mathrm{Fl}$ for plants indicated $100 \%$ choice of informants for treating specific ailments. Such species are Chromolaena odoratum, Ceriscoides campanulata, Oroxylum indicum, Cuscuta reflexa, Averrhoa carambola, Cajanus cajan, Justicia adhatoda and Citrus aurantifolia. These are cultural bounded species and indicated their healing potential. 
Knowledge of ethnomedical plants

Table 3. Fidelity level (Fl) values of the frequently reported plants and their major uses.

\begin{tabular}{|c|c|c|c|c|}
\hline Scientific name & Ailments & $\begin{array}{c}\text { Number of } \\
\text { informants (Ip) }\end{array}$ & $\begin{array}{l}\text { Total informants } \\
\text { (Iu) }\end{array}$ & $\begin{array}{c}\text { Fidelity level } \\
(\%)\end{array}$ \\
\hline $\begin{array}{l}\text { Chromolaena } \\
\text { odoratum }\end{array}$ & $\begin{array}{l}\text { Cut and } \\
\text { wound }\end{array}$ & 32 & 32 & 100 \\
\hline $\begin{array}{l}\text { Ceriscoides } \\
\text { campanulata }\end{array}$ & Jaundice & 28 & 28 & 100 \\
\hline Oroxylum indicum & Jaundice & 27 & 27 & 100 \\
\hline Cuscuta reflexa & Jaundice & 25 & 25 & 100 \\
\hline Averrhoa carambola & Jaundice & 22 & 22 & 100 \\
\hline Cajanus cajan & Jaundice & 21 & 21 & 100 \\
\hline Justicia adhatoda & cough & 20 & 20 & 100 \\
\hline Citrus aurantifolia & Jaundice & 20 & 20 & 100 \\
\hline Cynodon dactylon & $\begin{array}{l}\text { Cut and } \\
\text { wound }\end{array}$ & 37 & 37 & 100 \\
\hline Syzygium cumini & Diabetes & 25 & 26 & 96.15 \\
\hline Mikania cordata & $\begin{array}{l}\text { Cut and } \\
\text { wound }\end{array}$ & 58 & 61 & 95.08 \\
\hline Ocimum sanctum & Cough & 53 & 60 & 88.33 \\
\hline Ocimum basilicum & Cough & 30 & 34 & 88.24 \\
\hline Litsea glutinosa & Dysentery & 64 & 79 & 81.01 \\
\hline $\begin{array}{l}\text { Andrographis } \\
\text { paniculata }\end{array}$ & Malaria & 34 & 50 & 68 \\
\hline $\begin{array}{l}\text { Clerodendrum } \\
\text { viscosum }\end{array}$ & $\begin{array}{l}\text { Stomach } \\
\text { ache }\end{array}$ & 30 & 48 & 62.5 \\
\hline Terminalia arjuna & Heart pain & 44 & 81 & 54.32 \\
\hline Centella asiatica & Dysentery & 40 & 76 & 52.63 \\
\hline Azadirahta indica & Dermatitis & 50 & 115 & 43.48 \\
\hline Sterculia villosa & impotence, & 27 & 68 & 39.71 \\
\hline Terminalia arjuna & Dysentery & 20 & 81 & 24.69 \\
\hline Azadirahta indica & Anthlemintic & 20 & 115 & 17.39 \\
\hline
\end{tabular}



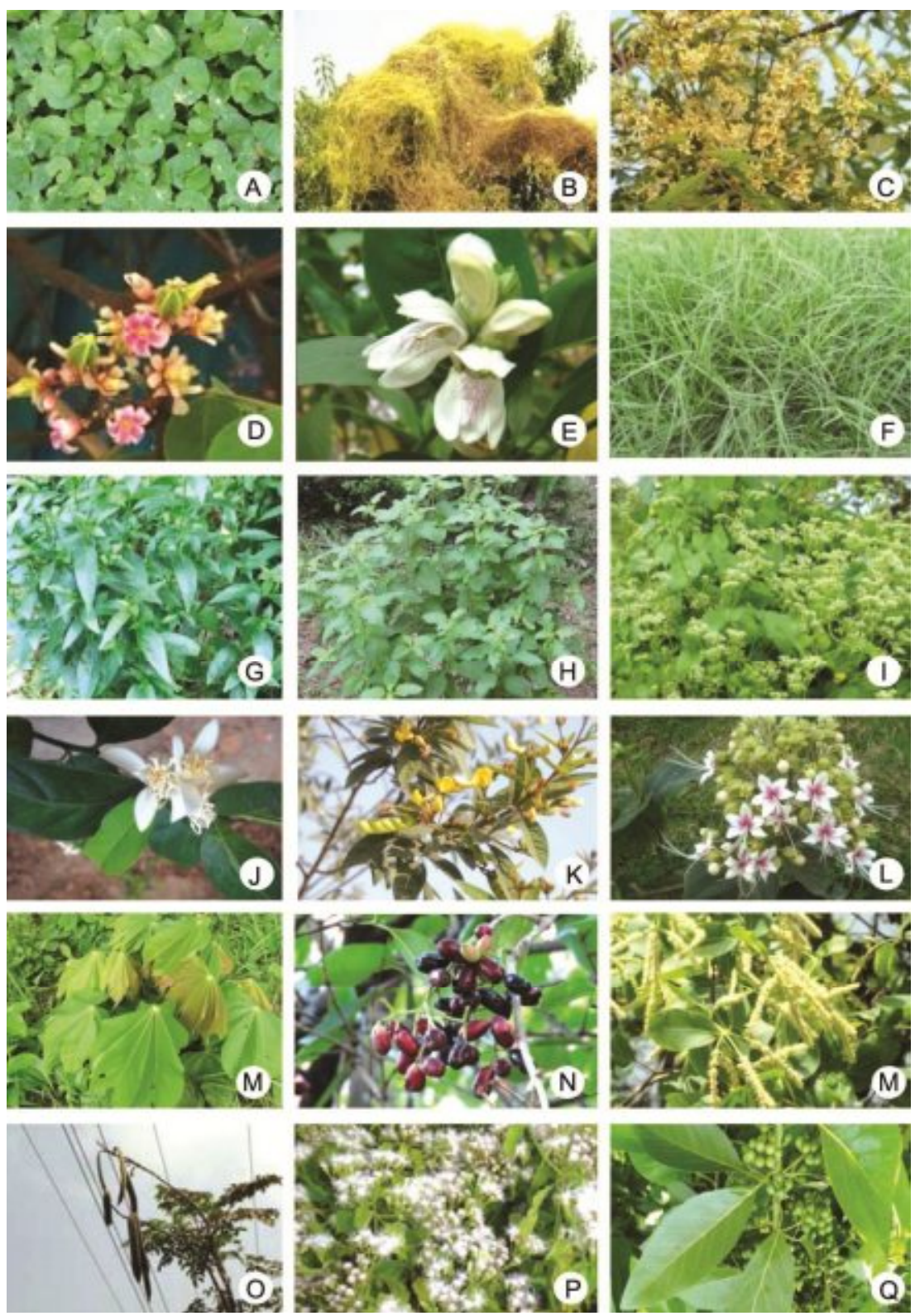

Plate 1. Images of most cited medicinal plants by the local people in the study area: $a$. Centella asiatica, b. Cuscuta reflexa, c. Azadirachta indica, d. Averrhoa carambola, e. Justicia adhatoda, f. Cynodon dactylon, g. Andrographis paniculata, h. Ocimum sanctum, i. Mikania cordata, j. Citrus aurantifolia, k. Cajanus cajan, 1. Clerodendrum viscosum, m. Sterculia villosa, n. Syzygium cumini, o. Terminalia arjuna, p. Oroxylum indicum, q. Chromolaena odoratum, r. Litsea glutinosa. 
PRK values varied from species to species as indicated in the Table 4. Litsea glutinosa scored top PRK value indicating very popular plant species in the study area and used for diarrhoea, dysentery and impotence. Mikania cordata, Ocimum sanctum, Azadirachta indica, Terminalia arjuna, Centella asiatica, Andrographis paniculata and Cynodon dactylon were also most cited species in the study area. These species are also well known medicinal plants in our country. The present analysis also confirmed their popularity among the local people of Lawachara national park. High PRK data of medicinal plants is the indication for further ethno-lead drug research to find active new drugs.

Table 4. Citation frequency of most cited medicinal plants.

\begin{tabular}{lllcc}
\hline Scientific name & Family & Ailments & Citation no. & $\begin{array}{c}\text { Percentage of } \\
\text { Respondents } \\
\text { Knowledge } \\
\text { (PRK\%) }\end{array}$ \\
\hline Litsea glutinosa & Lauraceae & Dysentery & 64 & 39.26 \\
Mikania cordata & Asteraceae & Cut and wound & 58 & 35.58 \\
Ocimum sanctum & Lamiaceae & Cough & 53 & 32.52 \\
Azadirachta indica & Meliacae & Dermatities & 50 & 30.67 \\
Terminalia arjuna & Caesalpiniacae & Heart pain & 44 & 26.99 \\
Centella asiatica & Clusiaceae & Dysentery & 40 & 24.54 \\
Andrographis paniculata & Acanthaceae & Malaria & 34 & 20.86 \\
Cynodon dactylon & Poaceae & Cut and wound & 34 & 20.86 \\
Chromolaena odoratum & Asteraceae & Cut wound & 32 & 19.63 \\
Clerodendrum viscosum & Verbenaceae & Stomach ache & 30 & 18.40 \\
Ocimum basilicum & Lamiaceae & Cough & 30 & 18.40 \\
Ceriscoides campanulata & Rubiaceae & Jaundice & 28 & 17.18 \\
Oroxylum indicum & Bignoniaceae & Jaundice & 27 & 16.56 \\
Sterculia villosa & Sterculiaceae & impotence, & 27 & 16.56 \\
Cuscuta reflexa & Cuscutaceae & Jaundice & 25 & 15.34 \\
Syzygium cumini & Myrtaceae & Diabetes & 25 & 15.34 \\
Averrhoa carambola & Averrhoaceae & Jaundice & 22 & 13.50 \\
Cajanus cajan & Fabaceae & Jaundice & 21 & 12.88 \\
Justicia adhatoda & Acanthaceae & cough & 20 & 12.27 \\
Azadirachta indica & Meliaceae & Anthlemintic & 20 & 12.27 \\
Citrus aurantifolia & Rutaceae & Jaundice & 20 & 12.27 \\
Terminalia arjuna & Combretaceae & Dysentery & 20 & 12.27 \\
\hline & & & & \\
\hline
\end{tabular}

Observations in the field, interviews and discussions with local people, a good number of threats to medicinal plants have been identified. The most serious threats are exotic timber species plantation in and around national park, fallow lands, homesteads, roadsides and even in the edges cultivated lands. Acacia auriculiformis, Acacia mangium, Eucalyptus camadulensis, Dalbergia sissoo, Laeucaena leucocephala, Swietenia mahagoni and Cassia siamea are most preferred plant species for plantation. According 
to local people perception such species are very selfish plants and they do not support native species under their canopy. Illegal logging and over exploitation from the national park vegetation are other threats to medicinal plants in the study area. Lack of awareness among the local people about impact of exotics on native plants and environment is another threat to medicinal plants. Availability of the modern medicines which promotes the negligence of use of herbal medicines among the local people in the study area is also threats to medicinal plants. Senior people with herbal knowledge do not like to share their knowledge with juniors. Due to sudden death of such people, herbal knowledge of the area lost forever.

A list of conservation measures is made based on present survey results and observations. Distribution map of all culturally important medicinal plant species in the study area could be made. Population status of such species across the habitats could be determined. Current rate of exploitation by local people could be calculated. If it seems that medicinal plants are vulnerable in the natural habitats, necessary measures could be taken for ex situ conservation. Awareness programs among the local influential persons who can make change could be created.

The present endeavor in and around Lawachara national park is very preliminary. It would be better if we could take more interviews with local people in the study area. Due to budget and time constrains, the present study is not well enough to draw sound conclusion. It needs further long term survey to further validate all ethnomedically important data for ethno-lead drug exploration research. In future, joint research in ethnobotany with pharmacognosy and phytochemistry are essential to confirm traditional knowledge of medicinal plants. The present quantitative evaluation of ethnobotanical data from in and around Lawachara national park probably is the first effort. The data indicated that the study area has plenty of medicinal plants (124 species) and diversity health care uses (53 ailments with 244 formularies) of such plants. Using modern mathematical tools Fic values on the uses of medicinal plants have been determined. The average Fic value for all ailment categories obtained 0.78 . Such value indicated that maximum people in the study area were well informed about the medicinal knowledge of plants and also showed agreement on the use of medicinal plants for such categories of ailments. Maximum Fic values (more than 0.80) obtained in case of Cut and wound, Jaundice, Respiratory related ailments, Diabetes, Diarrhoea and dysentery, Anthelmintic, Gastrointestinal complain, Impotence, Dermatitis and High blood pressure. Chromolaena odoratum, Ceriscoides campanulata, Oroxylum indicum, Cuscuta reflexa, Averrhoa carambola, Cajanus cajan, Justicia adhatoda and Citrus aurantifolia showed 100\% Fl values. Litsea glutinosa, Mikania cordata, Ocimum sanctum and Azadirahta indica were scored maximum PRK value. It is recommended that species showed high Fic, Fl and PRK values could be used for further ethno-lead phytochemical analysis to find active compounds to discover drugs from plants. As the medicinal plants and traditional knowledge in and around the park are in threatened condition, appropriate measures should be taken for sustainable conservation. For the community development, resource conservation, primary health care and economic growth such plants and knowledge can play an important role. 


\section{Acknowledgements}

The authors acknowledge the University Grants Commission (UGC) Bangladesh for partial financial aid for the research. The authors are also thankful to informants in the data collection process during the field works in and around Lawachara National Park. Cooperation from Local range office of Lawachara National Park is also acknowledged.

\section{References}

Ahmad, N. 1970. Working plan for the forests of the Sylhet division for the period 1963-64 to 1982-83, Working plan division 2, CTG.E.P. Dacca, East Pakistan Govt. Press, pp. 1-22.

Ahmed, Z.U., M.A. Hassan, Z.N.T. Begum, M. Khondker, S.M.H. Kabir, M. Ahmad, A.T.A Ahmed, A.K.A. Rahman and E.U. Haque, (Eds.) 2008a. Encyclopedia of Flora and Fauna of Bangladesh, Vol. 6. Angiosperms: Dicotyledons (Acanthaceae - Asteraceae). Asiatic Society of Bangladesh, Dhaka. pp. 1-408.

Ahmed, Z.U., M.A. Hassan, Z.N.T. Begum, M. Khondker, S.M.H. Kabir, M. Ahmad, A.T.A. Ahmed, A.K.A. Rahman and E.U. Haque, (Eds) 2008b. Encyclopedia of Flora and Fauna of Bangladesh, Vol. 12. Angiosperms: Monocotyledons (Orchidaceae Zingiberaceae). Asiatic Society of Bangladesh, Dhaka. pp. 1-552.

Ahmed, Z.U., M.A. Hassan, Z.N.T. Begum, M. Khondker, S.M.H. Kabir, M. Ahmad, A.T.A. Ahmed, A.K.A. Rahman and E.U. Haque,(Eds.) 2009b. Encyclopedia of Flora and Fauna of Bangladesh, Vol. 7. Angiosperms: Dicotyledons (Balsaminaceae Euphorbiaceae). Asiatic Society of Bangladesh, Dhaka. pp. 1-546.

Ahmed, Z.U., M.A. Hassan, Z.N.T. Begum, M. Khondker, S.M.H. Kabir, M. Ahmad, A.T.A. Ahmed,A.K.A. Rahman and E.U. Haque,(Eds.) 2009c. Encyclopedia of Flora and Fauna of Bangladesh, Vol. 8. Angiosperms: Dicotyledons (Fabaceae-Lythraceae). Asiatic Society of Bangladesh, Dhaka. pp. 1-478.

Ahmed, Z.U., M.A. Hassan, Z.N.T. Begum, M.Khondker, S.M.H. Kabir, M. Ahmad and A.T.A. Ahmed,(Eds.) 2009d. Encyclopedia of Flora and Fauna of Bangladesh, Vol. 9. Angiosperms: Dicotyledons (Magnoliaceae-Punicaceae). Asiatic Society of Bangladesh, Dhaka. pp. 1-488.

Ahmed, Z.U., M.A. Hassan, Z.N.T. Begum, M. Khondker, S.M.H. Kabir, M. Ahmad and A.T.A. Ahmed, (Eds.) 2009e.Encyclopedia of Flora and Fauna of Bangladesh, Vol. 10. Angiosperms: Dicotyledons (Ranunculaceae -Zygophyllaceae). Asiatic Society of Bangladesh, Dhaka. pp. 1-580.

Ahsan, M.F. 2000. Socio-Ecology of the Hoolock Gibbon (Hylobates hoolock) in Two Forests of Bangladesh. Field Research: 284-299.

Ahsan, M.M. 2007. Perceptions of tourism by indigenous communities living in and adjoining Lawachara National Park. In: Fox, J., Bushley, B.R, Dutt, S and Quazi, S.A. (eds.). Making conservation work: linking rural livelihoods and protected areas management in Bangladesh. East-West Centre and Nishorgo Program of the Bangladesh Forest department. pp. 131-148.

Alam, M. K. 1988. Annotated checklist of the woody flora of Sylhet forests. Bull. 5. Plant Taxonomy Series. Bangladesh Forest Research Institute, Chittagong. 153 pp.

Alam, M.K. 1992. Medical ethno-botany of the Marma tribe of Bangladesh. Economic Botany 46(3): 330-330.

Alam, M.K. 1998. Documentation of Ethnobiological Information. In: Banik, R.L., Alam, M.K., Peil, S. and Rastogi, A. (eds). Applied Ethnobotany. Bangladesh Forest Research Institute: Chittagong. pp. 28-29

Alam, M.K., J.U. Choudhury and M.A. Hassan, 1996. Some folk formularies from Bangladesh. Bangladesh J. Life Sci. 8(1): 49-63.

Alexiades, M.N. (ed.) 1996.Selected Guidelines for Ethno botanical Research: A Field Manual. 
The New York Botanical Garden, New York. 1-306 pp.

Borris, R P. 1999. Natural products research: perspectives from a major pharmaceutical company. $J$ Ethnopharmacol.51:29-38.

Breevot, P. 1998. The booming of US botanical market: A new overview of herbal gram. 44: 3346.

Canonizado, J.A. and S.M. Rahman, 1998. GOB/WB Forest resources management project technical assistance component: integrated forest management plan for the Sylhet forest division. Mandala Agricultural Development Corporation and Forest Department, Ministry of Environment and Forest, Dhaka, Bangladesh, pp. 1-4.

Chambers, R. 1994. Participatory Rural Appraisal (PRA): Analysis of experience. World Development 22(9): 1253-1268.

Friedman, J., Z. Yaniv, A. Dafni, and D. Palewitch, 1986. A preliminary classification of healing potential plants, based on a rational analysis of an ethno pharmacological field survey among Bedouins in the Negev Desert, Israel. Journal of ethno pharmacology. 16: 275287.

Green, 1990. IUCN Directory of South Asian protected areas. IUCN The World Conservation Union, Gland, Switzerland and Cambridge, UK, pp. 24-30.

Haque, T., M.Z. Uddin, M.L. Saha, M.A. Mazid and M.A. Hassan, 2014. Propagation, antibacterial activity and phytochemical properties of Litsea glutinosa (Lour.) C. B. Rob. Dhaka Univ. J. Biol. Sci. 23(2): 165-171.

Hassan, M.A. and M.S. Khan, 1986. Ethnobotanical record of Bangladesh-1: Plants used for healing fractured bones. J. Asiatic Soc. Bangladesh. (Sci.).12(1\&2): 33-39.

Hassan, M.A. and M.S. Khan, 1996. Ethnobotanical record of Bangladesh-2. Plants used for healing cuts and wounds. Bangladesh J. Plant Taxon. 3(2): 49-52.

Heinrich, M., A. Ankli, B. Frei, and C. Weimann, 1998. Medicinal plants in Mexico: healers consensus and cultural importance. Social Science and Medicine 47: 1859-1871.

Hooker, J.D. 1872-1897. Flora of British India 1-7. First Indian Reprint 1973 Bishen Singh Mahendra Pal Singh, Dehra Dun, India.

Hyland, B.P.M. 1972. A technique for collecting botanical specimens in rain forest. Flora Malesiana Bulletin. 26: 2038-2040.

Khan, I., N.M. Abdelsalam,. H. Fouad, A. Tariq, R. Ullah and M. Adnan, 2014. Evident-Based complementary and alternative medicine: Article ID 635371, p.21 Hindwai.

Khan, M.S., M.A.Hassan and M.Z. Uddin, 2002.Ethnobotanical survey in Rema-Kalenga Wildlife Sanctuary (Habiganj) in Bangladesh. Bangladesh J. Plant Taxon. 9(1): 51-60.

Martin, G.J. 1995. Ethnobotany: A Methods Manual. Chapman \& Hall, London. 1-268 pp.

Mia, M.M.K. and A.M. Huq, 1988. A preliminary ethno-botanical survey in the Jointiapur, Tamabil and Jafflong area, Sylhet, Bangladesh National Herbarium Bull. 3: 1-10.

Moerman, D.E. 1996. An analysis of the food plants and drug plants of native North America. $J$. Ethnopharmacol. 52:1-22.

Prain, D. 1903. Bengal Plants Vol. 1-2: 1-1013pp. First Indian Reprint 1963, Bishen Singh Mahendra Pal Singh Dehra Dun.

Riadh, S.M. 2007. Assessing the role of non-timber forest products in the livelihoods of communities living inside and outside of Lawachara National Park. In: Fox, J., Bushley, B.R, Dutt, S. and Quazi, S.A. (eds.). Making conservation work: linking rural livelihoods and protected areas management in Bangladesh. East-West Centre and Nishorgo Program of the Bangladesh Forest department. pp. 36-49.

Rizvi, S.N.H. 1970. East Pakistan District Gazetteers for Sylhet. Government of East Pakistan Services and General Administration Department, Dhaka, pp. 5-6.

Schultes, R E. 1978. The kingdom of plants. In: Thomson W A R, editor. Medicines from the Earth. New York, N.Y: McGraw-Hill Book Co.; p. 208.

Setzer, M.S., J.S. Werka, A.K. Irvine, B.R. Jackes and W.N. Setzer, 2006. Biological activity of rain forest plant extracts from far north Queensland, Australia. In: Williams L.A.D. (ed.). 
Biologically active natural products for $21^{\text {st }}$ century. Trivandrum, India: Research Signpost: p 21-46.

Siddiqui, K.U., M.A. Islam, Z.U. Ahmed, Z.N.T. Begum, M.A. Hassan, M. Khondker, M.M Rahman, S.M.H. Kabir, M.Ahmad, A.T.A.Ahmed, A.K.A. RahmanandE.U.Haque, (Eds) 2007c. Encyclopedia of Flora and Fauna of Bangladesh, Vol.11. Angiosperms: Monocotyledons (Agavaceae-Najadaceae). Asiatic Society of Bangladesh, Dhaka. pp.1399.

Stevens, P.R. 1986. Land classification and soil suitability for plantation in Sylhet division. Working Paper No. 26 (UNDP/FAO) Project BGD/79/017, 21 pp.

Trotter, R.T. and M.H. Logan, 1986. Informant census: A new approach for identifying potentially effective medicinal plants. Page 91-112 in L.N. Etkin, ed., Plants in indigenous medicine and diet. Redgrave, Bedford Hill, New York.

Uddin, M.Z and M.A Hassan, 2014. Determination of informant consensus factor of ethnomedicinal plants used in kalenga forest, Bangladesh. Bangladesh j. plant taxon. 21(1): 83-91.

Uddin, M.Z. and M.A. Hassan, 2004. Flora of Rema-Kalenga Wildlife Sanctuary. IUCN Bangladesh Country Office, Dhaka, Bangladesh, vi+120pp.

Uddin, M.Z., M.A. Hassan, M.M. Rahman, and M.K. Arefin, 2012.Ethno-medico-botanical study in Lawachara National Park, Bangladesh. Bangladesh J. Bot.41(1): 97-104.

Uddin, M.Z. and S. Roy, 2007. Collection and Management of Selected Medicinal Plants in RemaKalenga Wildlife Sanctuary.In: Making Conservation Work: Linking rural livelihoods and protected area management in Bangladesh edited by Fox, J. Bushley, B. R., Dutta S. and Quazi, S. A. 2007. Monograph of East-West Center, Hawaii, USA: 66-83pp.

Uddin, M.Z. S. Roy, M.A. Hassan and M.M. Rahman, 2008. Medicobotanical report on the Chakma people of Bangladesh. Bangladesh J. Plant Taxon.15 (1): 67-72

Uddin, M.Z., M.A. Hassan and M. Sultana, 2006. Ethnobotanical survey of medicinal plants in Phulbari Upazila of Dinajpur District, Bangladesh. Bangladesh J. Plant Taxon. 12(1): 63 68.

Uddin, M.Z., M.S. Khan and M.A. Hassan, 2001. Ethno medical plants records of Kalenga forest range (Habiganj), Bangladesh for malaria, jaundice, diarrhoea and dysentery. Bangladesh J. Plant Taxon. 8(1): 101-104.

Uddin, S.N. 2006. Traditional uses of ethnomedicinal plants of the Chittagong Hill Tracts. Bangladesh National Herbarium, Dhaka. 879p.

Uddin, S.N., M.Z. Uddin, M.A. Hassan and M.M. Rahman, 2004.Preliminary ethno-medical plant survey in Khagrachari district, Bangladesh. Bangladesh J. Plant Taxon. 11(2): 39-48.

http://www.usaid.gov/bd/files/NSPManagement_Plans_for_LNP.pdf

Wright, C.W. 2005. Plant derived antibacterial agents: new leads and challenges, Phytochemistry Reviews. 4 (1): pp. 55-61.

USAID, 2006. Management Plans for Lawachara National Park. International Resources Group (IRG), Connecticut, Washington. $207 \mathrm{pp}$.

Yusuf, M., M.A. Rahman, J.U. Choudhury and J. Begum, 2002.Indigenous knowledge about the use of Zingibers in Bangladesh. J. Econ. Taxon. Bot. 26(3): 566-570.

Yusuf, M., M.A. Wahab, J.U. Choudhury and J. Begum, 2006. Ethno-medico-botanical knowledge from Kaulkhali proper and Betunia of Rangamati district. Bangladesh J. Plant Taxon. 13(1): 55-61. 